\title{
Macrolactonization via Ti(IV)-Mediated Epoxy-Acid Coupling: A Total Synthesis of (-)-Dactylolide [and Zampanolide]
}

\author{
Thomas R. Hoye, ${ }^{*}$ and $\mathrm{Min} \mathrm{Hu}$ \\ 207 Pleasant Street, SE, Department of Chemistry, \\ University of Minnesota, Minneapolis, MN 55455 \\ hoye@chem.umn.edu
}

\section{Supporting Information}

Spectroscopic characterization data for compounds 1-15, 18, 19, and 21.

\section{General Information.}

${ }^{1} \mathrm{H}$ and ${ }^{13} \mathrm{C}$ NMR spectra were recorded on a Varian VI-500, VI-300, or VXR-300 spectrometer. Proton chemical shifts are referenced to TMS $(0.00 \mathrm{ppm})$ for spectra obtained in $\mathrm{CDCl}_{3}$. Non-first order multiplets are identified as "nfom." Carbon chemical shifts are referenced to $\mathrm{CDCl}_{3}(77.00 \mathrm{ppm})$. Infrared (IR) spectra were obtained in a Prospect MIDAC FT-IR spectrometer. High resolution mass spectra were recorded either on a Finnigan FTMS 2001 or Bruker Biotof II instrument. Optical rotations were measured on a JASCO-DIP-370 polarimeter using a $3.5 \mathrm{~mm}$ i.d. x $50 \mathrm{~mm}$ 1. cell. Combustion analyses were performed by M-H-W Laboratories, Phoenix, AZ. 


\section{(-)-(1S,2E,5S,8E,10Z,14E,17S)-3,11-Dimethyl-19-methylene-7,13-dioxo-6,21-}

\section{dioxabicyclo[15.3.1]heneicosa-2,8,10,14-tetraene-5-carboxaldehyde [(-)-Dactylolide (1)]}

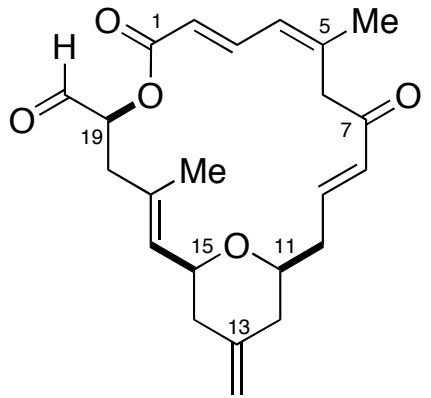

1

To a stirred benzene ( $3 \mathrm{~mL})$ solution of diol $15(4.1 \mathrm{mg}, 0.01 \mathrm{mmol})$ was added $\mathrm{Pb}(\mathrm{OAc})_{4}(16$ $\mathrm{mg}, 0.036 \mathrm{mmol}$ ) at room temperature. After consumption of starting material (tlc, $\sim 45 \mathrm{~min}$ ), the reaction mixture was directly load onto a silica-gel column and eluted with hexane/EtOAc (6:1 to $2: 1)$ to provide a $4: 1$ mixture of dactylolide [(-)-1] and its hydrate $(3.8 \mathrm{mg}, 90 \%)$ as a colorless oil.

${ }^{1} \mathbf{H}$ NMR (500 MHz, $\mathrm{CDCl}_{3}$ ): $\square 9.67$ (s, 1H, CHO), 7.64 (dd, J = 14.9 and $11.6 \mathrm{~Hz}, 1 \mathrm{H}, \mathrm{H}-3$ ), 6.85 (ddd, $\mathrm{J}=16.2,8.6$, and $6.0 \mathrm{~Hz}, 1 \mathrm{H}, \mathrm{H}-9), 6.16$ (d, J = 11.6 Hz, 1H, H-4), 6.01 (d, J = 16.2 Hz, 1H, H-2), 5.97 (d, J = 14.9 Hz, 1H, H-8), 5.32 (dd, J = 11.6 and 2.4 Hz, 1H, H-19), 5.24 (d, J $=8.0 \mathrm{~Hz}, 1 \mathrm{H}, \mathrm{H}-16), 4.75\left(\mathrm{~s}, 2 \mathrm{H}, \mathrm{H}_{2} \mathrm{C}=\mathrm{C}-13\right), 3.97(\mathrm{ddd}, \mathrm{J}=11.1,8.0$, and $2.7 \mathrm{~Hz}, 1 \mathrm{H}, \mathrm{H}-15)$, $3.96\left(\mathrm{~d}, \mathrm{~J}=14.3 \mathrm{~Hz}, 1 \mathrm{H}, \mathrm{H}_{\mathrm{a}}-6\right), 3.33$ (dddd, $\mathrm{J}=11.9$, 9.6, 2.7, and 2.7 Hz, 1H, H-11), $3.23(\mathrm{~d}, \mathrm{~J}=$ 14.3 Hz, 1H, H $\left.\mathrm{b}_{\mathrm{b}}-6\right), 2.55$ (d, J = 13.9 Hz, 1H, $\left.\mathrm{H}_{\mathrm{a}}-18\right), 2.32$ (d, J = 14.1 Hz, 1H, $\left.\mathrm{H}_{\mathrm{b}}-18\right), 2.40-2.27$ $(\mathrm{m}, 2 \mathrm{H}), 2.18\left(\operatorname{app} \mathrm{d}, \mathrm{J}=13.3 \mathrm{~Hz}, 1 \mathrm{H}, \mathrm{H}_{\mathrm{b}}-18\right), 2.11\left(\operatorname{app} \mathrm{d}, \mathrm{J}=12.9 \mathrm{~Hz}, 1 \mathrm{H}, \mathrm{H}_{\mathrm{eq}}-14\right), 1.96(\mathrm{t}, \mathrm{J}=$ 12.4Hz, 1H, H $\mathrm{ax}^{-12)}, 1.95$ (m, 1H, H), 1.86 (s, 3H, Me-C-5), and 1.72 (s, 3H, Me-C-17).

${ }^{13}$ C NMR (125 MHz, $\left.\mathrm{CDCl}_{3}\right)$ : $\square$ 199.1, 197.4, 166.3, 146.0, 144.0, 143.5, 140.4, 131.5, 130.9, $130.5,125.6,119.8,109.3,76.5,75.7,75.3,44.9,40.8,40.4,39.71,39.67,24.1$, and 16.0.

IR (neat): 3428, 2935, 2892, 1718, 1700, 1653, 1635, and $1281 \mathrm{~cm}^{-1}$.

HRMS (ESI +PEG) Calcd for $\mathrm{C}_{23} \mathrm{H}_{28} \mathrm{O}_{5} \mathrm{Na}(\mathrm{M}+\mathrm{Na})^{+}:$407.1829, found: 407.1831; Calcd for $\mathrm{C}_{23} \mathrm{H}_{30} \mathrm{O}_{6} \mathrm{Na}\left(\mathrm{M}+\mathrm{H}_{2} \mathrm{O}+\mathrm{Na}\right)^{+}: 425.1935$, found: 425.1927; Calcd for $\mathrm{C}_{24} \mathrm{H}_{32} \mathrm{O}_{6} \mathrm{Na}$ $(\mathrm{M}+\mathrm{MeOH}+\mathrm{Na})^{+}:$439.2091, found: 439.2099.

$[\square]_{D}^{23^{\circ} \mathrm{C}}=-127.8^{\circ}$ and $128.9^{\circ}, \mathrm{c}=0.39$ and 0.26 , respectively, in $\mathrm{MeOH}$. 
(2E,5E)-7-\{[(1,1-Dimethylethyl)diphenylsilyl]oxy\}-3-methyl-2,5-heptadienal (2)

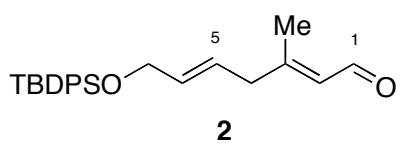

${ }^{1} \mathbf{H}$ NMR (500 MHz, $\mathrm{CDCl}_{3}$ ): $\square 10.00$ (d, J = 7.5 Hz, 1H, H-1), 7.68-7.66 (m, 4H, ArH), 7.45-

$7.36(\mathrm{~m}, 6 \mathrm{H}, \mathrm{ArH}), 5.89(\mathrm{dq}, \mathrm{J}=8.0$ and $1.0 \mathrm{~Hz}, 1 \mathrm{H}, \mathrm{H}-2), 5.71-5.62$ (m, 2H, H-5 and H-6), 4.21-4.19 (nfom, 2H, H-7), 2.93-2.90 (nfom, 2H, H-4), 2.14 (d, J = 1.0 Hz, 3H, Me), and 1.06 (s, $9 \mathrm{H}, t-\mathrm{Bu}-\mathrm{Si})$.

${ }^{13}$ C NMR (125 MHz, $\left.\mathrm{CDCl}_{3}\right)$ : $\square$ 191.3, 162.3, 133.6, 135.5, 133.0, 129.7, 129.6, 127.7, 124.9, 64.0, 43.1, 26.8, 19.2, and 17.5.

IR (neat): 3071, 2957, 2932, 2858, 1725, 1675, and $1428 \mathrm{~cm}^{-1}$.

HRMS (ESI) Calcd for $\mathrm{C}_{24} \mathrm{H}_{30} \mathrm{O}_{2} \mathrm{SiNa}(\mathrm{M}+\mathrm{Na})^{+}: 401.1912$, found: 401.1907 .

TLC $R_{\mathrm{f}}=0.4$, hexanes : ethyl acetate $=6: 1$.

\section{(-)-(3S)-\{5-[(Trimethylsilyl)methyl]-3-[(trimethylsilyl)oxy]hex-5-enyl\} 2,2-}

dimethylpropanoate (3)

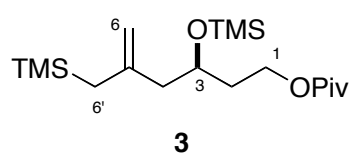

${ }^{1} \mathbf{H}$ NMR (500 MHz, $\mathrm{CDCl}_{3}$ ): $\square 4.62\left(\mathrm{ddd}, \mathrm{J}=2.0,1.0\right.$, and $\left.1.0 \mathrm{~Hz}, 1 \mathrm{H}, \mathrm{H}_{\mathrm{a}}-6\right), 4.59$ (ddd, J = 2.5, 1.0, and $\left.1.0 \mathrm{~Hz}, 1 \mathrm{H}, \mathrm{H}_{\mathrm{b}}-6\right), 4.20\left(\mathrm{ddd}, \mathrm{J}=11.0,6.0\right.$, and $\left.5.0 \mathrm{~Hz}, 1 \mathrm{H}, \mathrm{H}_{\mathrm{a}}-1\right), 4.06$ (ddd, $\mathrm{J}=11.0$, 9.0, and $5.5 \mathrm{~Hz}, 1 \mathrm{H}, \mathrm{H}_{\mathrm{b}}-1$ ), 3.93 (dddd, $\mathrm{J}=8.5,7.5,6.0$, and $\left.4.0 \mathrm{~Hz}, 1 \mathrm{H}, \mathrm{H}-3\right), 2.19$ (ddd, $\mathrm{J}=$ 13.5, 6.0, and $\left.1.0 \mathrm{~Hz}, 1 \mathrm{H}, \mathrm{H}_{\mathrm{a}}-4\right), 2.07$ (ddd, $\mathrm{J}=14.0,7.0$, and, $\left.1.0 \mathrm{~Hz}, 1 \mathrm{H}, \mathrm{H}_{\mathrm{b}}-4\right), 1.88$ (ddd, $\mathrm{J}=$ 14.5, 9.0, 6.0, and 3.5 Hz, 1H, $\mathrm{H}_{\mathrm{a}}-2$ ), 1.61 (dddd, $\mathrm{J}=14.0,9.0,5.0$, and $5.0 \mathrm{~Hz}, 1 \mathrm{H}, \mathrm{H}_{\mathrm{b}}-2$ ), 1.53 $\left(\mathrm{dd}, \mathrm{J}=13.0\right.$ and $\left.1.0 \mathrm{~Hz}, 1 \mathrm{H}, \mathrm{H}_{\mathrm{a}}-6^{\prime}\right), 1.51\left(\mathrm{dd}, \mathrm{J}=13.0\right.$ and $\left.1.0 \mathrm{~Hz}, 1 \mathrm{H}, \mathrm{H}_{\mathrm{b}}-6^{\prime}\right), 1.20$ (s, $9 \mathrm{H}, t$-BuPv), 0.12 (s, 9H, Me-OTMS), and 0.03 (s, 9H, Me-TMS).

${ }^{13}$ C NMR (125 MHz, $\left.\mathrm{CDCl}_{3}\right): \square 178.4,143.8,110.1,68.0,61.3,46.8,38.7,36.0,27.2,27.1,-0.3$, and -1.4.

IR (neat): 2958, 1732, and $1481 \mathrm{~cm}^{-1}$.

HRMS (ESI) Calcd for $\mathrm{C}_{18} \mathrm{H}_{38} \mathrm{O}_{3} \mathrm{Si}_{2} \mathrm{Na}(\mathrm{M}+\mathrm{Na})^{+}: 381.2252$, found: 381.2255.

TLC $R_{\mathrm{f}}=0.6$, hexanes : ethyl acetate $=9: 1$.

[ㅁ] $]_{D}^{23^{\circ} \mathrm{C}}=-28.2^{\circ}, \mathrm{c}=1.65, \mathrm{CH}_{2} \mathrm{Cl}_{2}$. 
(-)-2-[(2R,6S)-6-[(1E,4E)-6-\{[(1,1-Dimethylethyl)diphenylsilyl]oxy\}-2-methyl-1,4-

hexadienyl]tetrahydro-4-methylene-2H-pyran-2-yl]ethyl 2,2-dimethylpropanoate (4)

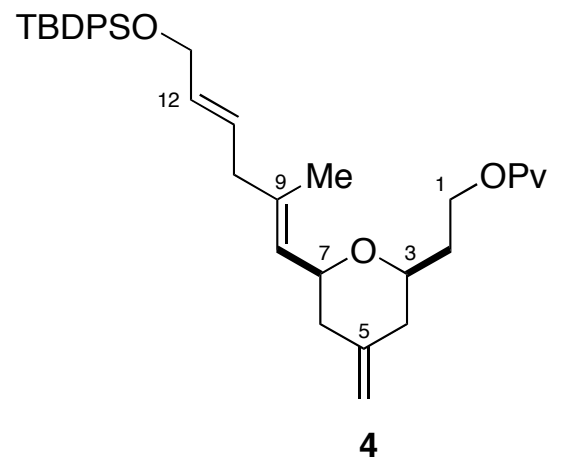

${ }^{1} \mathbf{H}$ NMR (500 MHz, $\left.\mathrm{CDCl}_{3}\right): \square 7.70-7.67$ (m, 4H, ArH), $7.44-7.36$ (m, 6H, ArH), 5.66 (dtt, J $=15.0,7.0$, and $1.5 \mathrm{~Hz}, 1 \mathrm{H}, \mathrm{H}-11), 5.58(\mathrm{dtt}, \mathrm{J}=15.0,5.0$, and $1.0 \mathrm{~Hz}, 1 \mathrm{H}, \mathrm{H}-12), 5.23(\mathrm{dq}, \mathrm{J}=$ 7.5 and $1.5 \mathrm{~Hz}, 1 \mathrm{H}, \mathrm{H}-8), 4.75\left(\mathrm{t}, \mathrm{J}=1.5 \mathrm{~Hz}, 2 \mathrm{H}, \mathrm{H}_{2} \mathrm{C}=\mathrm{C}-5\right), 4.18\left(\mathrm{~m}, 4 \mathrm{H}, \mathrm{H}_{\mathrm{a}, \mathrm{b}}-13\right.$ and $\left.\mathrm{H}_{\mathrm{a}, \mathrm{b}}-1\right)$, 3.98 (ddd, $\mathrm{J}=11.0,7.5$, and $2.0 \mathrm{~Hz}, 1 \mathrm{H}, \mathrm{H}-7$ ), 3.43 (dddd, $\mathrm{J}=10.5,8.5,5.0$, and $2.5 \mathrm{~Hz}, 1 \mathrm{H}, \mathrm{H}-$ 3), $2.73\left(\mathrm{~m}, 2 \mathrm{H}, \mathrm{H}_{\mathrm{a}, \mathrm{b}}-10\right), 2.20\left(\mathrm{~m}, 2 \mathrm{H}, \mathrm{H}_{\mathrm{eq}}-4\right.$ and $\left.\mathrm{H}_{\mathrm{eq}}-6\right), 2.05$ (app t, J = $\left.12.5 \mathrm{~Hz}, 1 \mathrm{H}, \mathrm{H}_{\mathrm{ax}}-6\right), 1.98$ (app t, $\mathrm{J}=12.5 \mathrm{~Hz}, 1 \mathrm{H}, \mathrm{H}_{\mathrm{ax}}-4$ ), 1.90 (dddd, $\mathrm{J}=14.0,8.0,6.0$, and $6.0 \mathrm{~Hz}, 1 \mathrm{H}, \mathrm{H}_{\mathrm{a}}-2$ ), 1.82 (dddd, $\mathrm{J}=14.0,7.0,7.0$, and $\left.4.5 \mathrm{~Hz}, 1 \mathrm{H}, \mathrm{H}_{\mathrm{b}}-2\right), 1.65(\mathrm{~d}, \mathrm{~J}=1.0 \mathrm{~Hz}, 3 \mathrm{H}, \mathrm{Me}-\mathrm{C}-7), 1.19$ (s, 9H, $t-\mathrm{Bu}-\mathrm{Pv}$ ), and 1.05 (s, 9H, $t$-Bu-Si).

${ }^{13}$ C NMR (125 MHz, $\left.\mathrm{CDCl}_{3}\right): \square 178.4,144.3,138.7,135.5,133.8,130.9,129.5,128.1,127.6$, $125.9,108.7,75.5,75.0,64.4,61.1,42.1,40.8,40.5,38.6,35.2,27.2,26.8,19.2$, and 16.9.

IR (neat): 2959, 2932, 2858, 1728, and $1428 \mathrm{~cm}^{-1}$.

Comb. Anal.: Calcd for $\mathrm{C}_{34} \mathrm{H}_{50} \mathrm{O}_{4} \mathrm{Si}: \mathrm{C}, 75.22 ; \mathrm{H}, 8.77 \%$. Found: C, 74.94; $\mathrm{H}, 8.80 \%$.

TLC $\mathrm{R}_{\mathrm{f}}=0.55$, hexanes : ethyl acetate $=1: 1$.

[ㅁ] $]_{D}^{23^{\circ} \mathrm{C}}=-9.4^{\circ}, \mathrm{c}=1.34, \mathrm{CH}_{2} \mathrm{Cl}_{2}$. 
(+)-2-[(2R,6S)-6-[(1E,4E)-6-[[(1,1-Dimethylethyl)diphenylsilyl]oxy]-2-methyl-1,4-

hexadienyl]tetrahydro-4-methylene-2H-pyran-2-yl]ethanal (5)

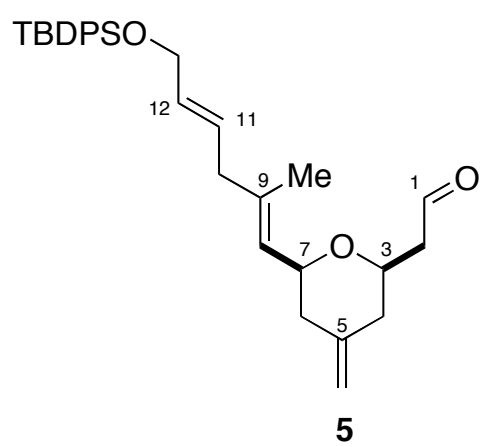

${ }^{1} \mathbf{H}$ NMR $\left(500 \mathrm{MHz}, \mathrm{CDCl}_{3}\right): \square 9.81(\mathrm{t}, \mathrm{J}=2.0 \mathrm{~Hz}, 1 \mathrm{H}, \mathrm{H}-1), 7.69-7.67$ (m, 4H, Ar-H), $7.44-$ $7.36(\mathrm{~m}, 6 \mathrm{H}, \mathrm{ArH}), 5.66(\mathrm{dtt}, \mathrm{J}=15.5,6.5$, and $1.5 \mathrm{~Hz}, 1 \mathrm{H}, \mathrm{H}-11), 5.58(\mathrm{dt}, \mathrm{J}=15.0$ and $4.5 \mathrm{~Hz}$, 1H, H-12), 5.22 (dq, J = 8.0 and $1.0 \mathrm{~Hz}, \mathrm{H}-8$ ), 4.78 (app t, $\left.\mathrm{J}=1.5 \mathrm{~Hz}, 2 \mathrm{H}, \mathrm{H}_{2} \mathrm{C}=\mathrm{C}-5\right), 4.17$ (m, $\left.2 \mathrm{H}, \mathrm{H}_{\mathrm{a}, \mathrm{b}}-13\right), 4.06$ (ddd, $\mathrm{J}=11.0,7.5$, and $2.5 \mathrm{~Hz}, 1 \mathrm{H}, \mathrm{H}-7$ ), 3.88 (dddd, $\mathrm{J}=12.5,7.0,4.5$, and $2.0 \mathrm{~Hz}, 1 \mathrm{H}, \mathrm{H}-3), 2.77-2.70\left(\mathrm{~m}, 2 \mathrm{H}, \mathrm{H}_{\mathrm{a}, \mathrm{b}}-10\right), 2.71$ (ddd, $\mathrm{J}=16.5,7.5$, and $\left.2.5 \mathrm{~Hz}, 1 \mathrm{H}, \mathrm{H}_{\mathrm{a}}-2\right)$, 2.54 (ddd, $\mathrm{J}=16.5,5.0$, and $2.0 \mathrm{~Hz}, 1 \mathrm{H}, \mathrm{H}_{\mathrm{b}}-2$ ), 2.27 (ddd, $\mathrm{J}=13.5,2.0$, and $2.0 \mathrm{~Hz}, 1 \mathrm{H}, \mathrm{H}_{\mathrm{eq}}-4$ ), 2.20 (ddd, $\mathrm{J}=13.5,2.0$, and $\left.1.5 \mathrm{~Hz}, 1 \mathrm{H}, \mathrm{H}_{\mathrm{eq}}-6\right), 2.09-1.99\left(2 \mathrm{H}, \mathrm{m}, \mathrm{H}_{\mathrm{ax}}-4\right.$ and $\left.\mathrm{H}_{\mathrm{ax}}-6\right), 1.65(\mathrm{~d}, \mathrm{~J}=$ $1.5 \mathrm{~Hz}, 3 \mathrm{H}, \mathrm{Me}-\mathrm{C}-9$ ), and 1.05 (s, 9H, $t$-Bu-Si).

${ }^{13}$ C NMR (125 MHz, $\left.\mathrm{CDCl}_{3}\right)$ : $\square 201.0,143.4,138.9,135.5,133.8,130.9,129.6,128.0,127.6$, 125.6, 109.5, 75.8, 73.3, 64.4, 49.7, 42.1, 40.5, 40.2, 26.8, 19.2, and 17.0.

IR (neat): 3069, 2931, 2857, 1725, and $1427 \mathrm{~cm}^{-1}$.

HRMS (CI) Calcd for $\mathrm{C}_{31} \mathrm{H}_{41} \mathrm{O}_{3} \mathrm{Si}(\mathrm{M}+\mathrm{H})^{+}$: 489.2819, found: 489.2834 .

TLC $\mathrm{R}_{\mathrm{f}}=0.6$, hexanes : ethyl acetate $=3: 1$.

$[\mathrm{G}]_{D}^{23^{\circ} \mathrm{C}}=+11.4^{\circ}, \mathrm{c}=1.55, \mathrm{CH}_{2} \mathrm{Cl}_{2}$. 
(-)-(1,1-Dimethylethyl)diphenyl[(2E,5E)-6-[(2S,6S)-6-[(2E)-3-iodo-2-propenyl]-tetrahydro4-methylene-2H-pyran-2-yl]-5-methyl-2,5-hexadienyl]oxy]silane (6a)

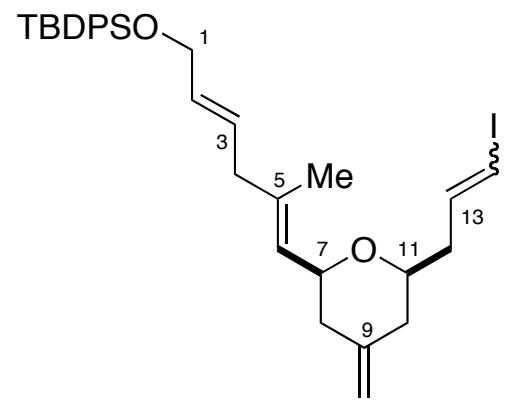

$6 \mathbf{a}$

${ }^{1} \mathbf{H}$ NMR (500 MHz, $\mathrm{CDCl}_{3}$ ): $\square 7.70-7.67$ (m, 4H, $\left.\mathrm{ArH}\right), 7.44-7.36(\mathrm{~m}, 6 \mathrm{H}, \mathrm{ArH}), 6.57$ (dt, J = 15.0 and $7.5 \mathrm{~Hz}, 1 \mathrm{H}, \mathrm{H}-14), 6.36-6.29$ (m, 2H, H-13 and H-14, Z-isomer), 6.10 (dt, J = 14.5 and $1.5 \mathrm{~Hz}, 1 \mathrm{H}, \mathrm{H}-14$ ), 5.67 (dtt, $\mathrm{J}=15.0,8.0$, and $1.5 \mathrm{~Hz}, 1 \mathrm{H}, \mathrm{H}-2), 5.59$ (dt, $\mathrm{J}=15.0$ and $5.0 \mathrm{~Hz}$, $1 \mathrm{H}, \mathrm{H}-3), 5.23(\mathrm{dq}, \mathrm{J}=8.0$ and $1.5 \mathrm{~Hz}, 1 \mathrm{H}, \mathrm{H}-6), 4.75$ (app t, $\left.\mathrm{J}=1.5 \mathrm{~Hz}, 2 \mathrm{H}, \mathrm{H}_{2} \mathrm{C}=\mathrm{C}-9\right), 4.18$ $\left(\operatorname{app~dq}, \mathrm{J}=5.0\right.$ and $\left.1.0 \mathrm{~Hz}, 2 \mathrm{H}, \mathrm{H}_{\mathrm{a}, \mathrm{b}}-1\right), 4.00(\mathrm{ddd}, \mathrm{J}=10.5,7.5$, and $2.5 \mathrm{~Hz}, 1 \mathrm{H}, \mathrm{H}-7), 3.46$ (dddd, $\mathrm{J}=11.1,6.0,6.0$, and $2.0 \mathrm{~Hz}, 1 \mathrm{H}, \mathrm{H}-11, \mathrm{Z}$-isomer), 3.37 (dddd, $\mathrm{J}=11.0,6.0$, 6.0, and 2.0 $\mathrm{Hz}, 1 \mathrm{H}, \mathrm{H}-11), 2.73$ (m, 2H, $\left.\mathrm{H}_{\mathrm{a}, \mathrm{b}}-4\right), 2.41$ (t, J = 6.0 Hz, 2H, $\mathrm{H}_{\mathrm{a}, \mathrm{b}}-12$, Z-isomer), 2.36 (dddd, J $=15.0,7.0,6.5$, and $\left.1.5 \mathrm{~Hz}, 1 \mathrm{H}, \mathrm{H}_{\mathrm{a}}-12\right), 2.28-2.14\left(\mathrm{~m}, 3 \mathrm{H}, \mathrm{H}_{\mathrm{eq}}-8, \mathrm{H}_{\mathrm{eq}}-10\right.$, and $\left.\mathrm{H}_{\mathrm{b}}-12\right), 2.03$ (app t, $\left.\mathrm{J}=12.5 \mathrm{~Hz}, 1 \mathrm{H}, \mathrm{H}_{\mathrm{ax}}-8\right), 1.93\left(\operatorname{app} \mathrm{t}, \mathrm{J}=12.5 \mathrm{~Hz}, 1 \mathrm{H}, \mathrm{H}_{\mathrm{ax}}-10\right), 1.66(\mathrm{~d}, \mathrm{~J}=1.5 \mathrm{~Hz}, 3 \mathrm{H}$, Me-C-5), and $1.06(\mathrm{~s}, 9 \mathrm{H}, t$-BuSi).

${ }^{13}$ C NMR (125 MHz, $\left.\mathrm{CDCl}_{3}\right)$ : $\square$ 144.0, 142.2, 138.4, 135.5, 133.7, 130.8, 129.5, 128.00,127.5, $125.8,108.9,76.8,76.55,75.66,64.3,42.5,42.1,40.7,39.8,26.8,19.2$, and 16.98.

Distinct resonance from cis-isomer: 144.1, 137.5, 128.02, 125.9, 108.8, 84.3, 76.59, 75.73, 41.3, 39.9, and 16.96 .

IR (neat): $3071,2932,2856,1652$, and $1428 \mathrm{~cm}^{-1}$.

HRMS (FABMS, PEG + NaI) Calcd for $\mathrm{C}_{32} \mathrm{H}_{41} \mathrm{IO}_{2} \mathrm{SiNa}(\mathrm{M}+\mathrm{Na})^{+}:$635.1818, found: 635.1866. TLC $R_{\mathrm{f}}=0.43$, hexanes : ethyl acetate $=12: 1$. $[\square]_{D}^{23^{\circ} \mathrm{C}}=-3.1^{\circ}, \mathrm{c}=1.54, \mathrm{CH}_{2} \mathrm{Cl}_{2},(E: Z=4: 1)$. 
(-)-(1,1-Dimethylethyl)diphenyl[[(2E,5E)-6-[(2S,6S)-6-(2-propenyl)tetrahydro-4-methylene2H-pyran-2-yl]-5-methyl-2,5-hexadienyl]oxy]silane (6b)

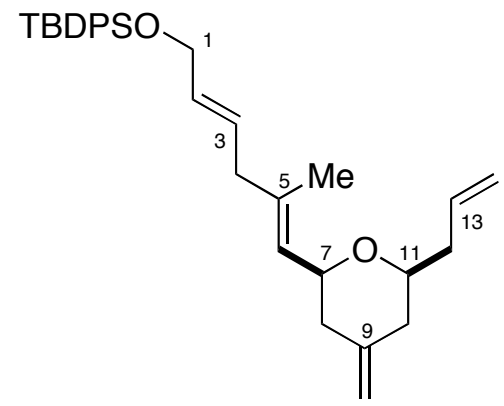

6b

${ }^{1}$ H NMR (500 MHz, $\mathrm{CDCl}_{3}$ ): $\square 7.69$ - 7.67 (m, 4H, ArH), 7.44 - 7.36 (m, 6H, ArH), 5.85 (dddd, $\mathrm{J}=17.0,10.5,7.5$, and $6.5 \mathrm{~Hz}, 1 \mathrm{H}, \mathrm{H}-13), 5.66(\mathrm{dtt}, \mathrm{J}=15.0,7.0$, and $1.5 \mathrm{~Hz}, 1 \mathrm{H}, \mathrm{H}-3), 5.58$ (dt, $\mathrm{J}=15.0$ and 5.0, 1H, H-2), $5.26(\mathrm{dq}, \mathrm{J}=7.5$ and $1.0 \mathrm{~Hz}, 1 \mathrm{H}, \mathrm{H}-6), 5.09$ (dddd, $\mathrm{J}=17.5,2.0,2.0$, and $\left.2.0 \mathrm{~Hz}, 1 \mathrm{H}, \mathrm{H}_{\text {cis }}-14\right), 5.05$ (dddd, $\mathrm{J}=10.5,2.5,1.0$, and $\left.1.0 \mathrm{~Hz}, 1 \mathrm{H}, \mathrm{H}_{\text {trans }}-14\right), 4.73(\operatorname{app~t}, \mathrm{J}=$ $\left.2.0 \mathrm{~Hz}, 2 \mathrm{H}, \mathrm{H}_{2} \mathrm{C}=\mathrm{C}-9\right), 4.17\left(\mathrm{~m}, 2 \mathrm{H}, \mathrm{H}_{\mathrm{a}, \mathrm{b}}-1\right), 4.01$ (ddd, $\mathrm{J}=11.0,8.0$, and $\left.3.0 \mathrm{~Hz}, 1 \mathrm{H}, \mathrm{H}-7\right), 3.37$ (dddd, $\mathrm{J}=11.5,6.5,6.5$, and $2.5 \mathrm{~Hz}, 1 \mathrm{H}, \mathrm{H}-11), 2.72\left(\mathrm{~m}, 2 \mathrm{H}, \mathrm{H}_{\mathrm{a}, \mathrm{b}}-4\right), 2.41(\mathrm{dtt}, \mathrm{J}=14.0,6.0$, and $\left.1.5 \mathrm{~Hz}, 1 \mathrm{H}, \mathrm{H}_{\mathrm{a}}-12\right), 2.27-2.21\left(\mathrm{~m}, 2 \mathrm{H}, \mathrm{H}_{\mathrm{b}}-12\right.$ and $\left.\mathrm{H}_{\mathrm{eq}}-10\right), 2.16$ (ddd, $\mathrm{J}=13.5,2.5$, and $1.5 \mathrm{~Hz}$, $1 \mathrm{H}, \mathrm{H}_{\mathrm{eq}}-8$ ), 2.04 (ddddd, $\mathrm{J}=13.0,11.0,1.5,1.5$, and $1.5 \mathrm{~Hz}, 1 \mathrm{H}, \mathrm{H}_{\mathrm{ax}}-8$ ), 1.93 (ddddd, $\mathrm{J}=13.0$, 11.0, 1.5, 1.5, and 1.5 Hz, 1H, $\left.\mathrm{H}_{\mathrm{ax}}-10\right), 1.65(\mathrm{~d}, \mathrm{~J}=1.0 \mathrm{~Hz}, 3 \mathrm{H}, \mathrm{Me}-\mathrm{C}-5)$, and $1.05(\mathrm{~s}, 9 \mathrm{H}, t-\mathrm{Bu}-$ $\mathrm{Si})$.

${ }^{13}$ C NMR (125 MHz, $\left.\mathrm{CDCl}_{3}\right)$ : $\square$ 144.7, 138.0, 135.5, 134.6, 133.8, 130.8, 129.5, 128.2, 127.6, $126.2,116.9,108.5,77.7,75.7,64.4,42.2,40.9,40.8,39.9,26.8,19.2$, and 16.9.

IR (neat): $3070,2930,2856,1653$, and $1428 \mathrm{~cm}^{-1}$.

Comb. Anal.: Calcd for $\mathrm{C}_{34} \mathrm{H}_{42} \mathrm{O}_{2} \mathrm{Si}$ : C, 78.96; H, 8.70\%. Found: C, 78.71; H, 8.68\%.

TLC $R_{\mathrm{f}}=0.6$, hexanes : ethyl acetate $=9: 1$.

$[\square]_{D}^{23^{\circ} \mathrm{C}}=-2.3^{\circ}, \mathrm{c}=1.07, \mathrm{CH}_{2} \mathrm{Cl}_{2}$. 


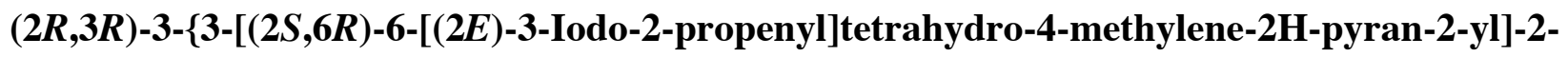
methyl-2-propenyl]oxirane methanol (7a)

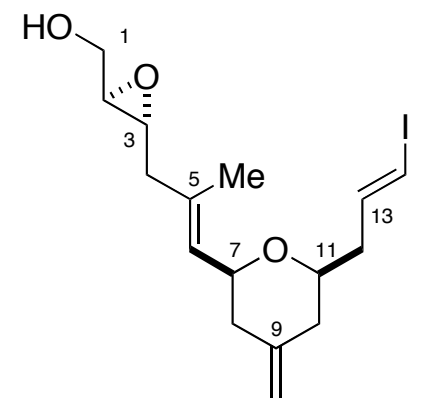

$7 a$

${ }^{1} \mathbf{H}$ NMR $\left(500 \mathrm{MHz}, \mathrm{CDCl}_{3}\right): \square 6.57(\mathrm{dt}, \mathrm{J}=15.5$ and $7.6 \mathrm{~Hz}, 1 \mathrm{H}, \mathrm{H}-13), 6.10(\mathrm{dt}, \mathrm{J}=15.5$ and $2.3 \mathrm{~Hz}, 1 \mathrm{H}, \mathrm{H}-14), 5.33$ (dq, J = 7.9 and $1.3 \mathrm{~Hz}, 1 \mathrm{H}, \mathrm{H}-6), 4.76$ (t, J = $1.8 \mathrm{~Hz}, 2 \mathrm{H}, \mathrm{H}_{2} \mathrm{C}=\mathrm{C}-9$ ), 4.02 (ddd, $\mathrm{J}=11.1,7.6$, and $2.7 \mathrm{~Hz}, 1 \mathrm{H}, \mathrm{H}-7$ ), 3.94 (ddd, $\mathrm{J}=12.7,5.5$, and $2.6 \mathrm{~Hz}, 1 \mathrm{H}, \mathrm{H}_{\mathrm{a}}-1$ ), 3.67 (ddd, $\mathrm{J}=12.7,7.7$, and $4.2 \mathrm{~Hz}, 1 \mathrm{H}, \mathrm{H}_{\mathrm{b}}-1$ ), 3.38 (dddd, $\mathrm{J}=11.3,6.6,5.8$, and $2.1 \mathrm{~Hz}, 1 \mathrm{H}, \mathrm{H}-$ 11), 3.08 (ddd, $\mathrm{J}=5.7,5.7$, and $2.3 \mathrm{~Hz}, 1 \mathrm{H}, \mathrm{H}-3$ ), 2.96 (ddd, $\mathrm{J}=4.1,2.5$, and $2.5 \mathrm{~Hz}, 1 \mathrm{H}, \mathrm{H}-2$ ), 2.38-2.17 (m, 6H, $\mathrm{H}_{\mathrm{a}, \mathrm{b}}-4, \mathrm{H}_{\mathrm{a}, \mathrm{b}}-12, \mathrm{H}_{\mathrm{eq}}-8$, and $\left.\mathrm{H}_{\mathrm{eq}}-10\right), 2.05$ (app t, J = 12.2 Hz, $1 \mathrm{H}, \mathrm{H}_{\mathrm{ax}}-8$ ), 1.93 (app t, $\left.\mathrm{J}=12.2 \mathrm{~Hz}, 1 \mathrm{H}, \mathrm{H}_{\mathrm{ax}}-10\right), 1.76(\mathrm{~d}, \mathrm{~J}=1.3 \mathrm{~Hz}, 3 \mathrm{H}, \mathrm{Me}-\mathrm{C}-5)$, and $1.64(\mathrm{dd}, \mathrm{J}=7.4$ and 5.5 $\mathrm{Hz}, 1 \mathrm{H}, \mathrm{OH})$.

${ }^{13} \mathrm{C}$ NMR (125 MHz, $\left.\mathrm{CDCl}_{3}\right): \square 143.7,142.2,135.3,127.8,109.1,76.9,76.6,75.4,61.4,58.2$, 54.4, 42.4, 41.4, 40.5, 39.7, and 17.4.

IR (neat): 3440, 2981, 2938, 2894, 1652, and $1423 \mathrm{~cm}^{-1}$.

HRMS (MSFAB, PEG + NaI) Calcd for $\mathrm{C}_{16} \mathrm{H}_{23} \mathrm{IO}_{3} \mathrm{Na}(\mathrm{M}+\mathrm{Na})^{+}: 413.0590$, found: 413.0597 .

TLC $\mathrm{R}_{\mathrm{f}}=0.38$, hexanes : ethyl acetate $=1: 1$.

$[\text { [] }]_{D}^{23^{\circ} \mathrm{C}}=+17.1^{\circ}, \mathrm{c}=2.5, \mathrm{CH}_{2} \mathrm{Cl}_{2}$. 


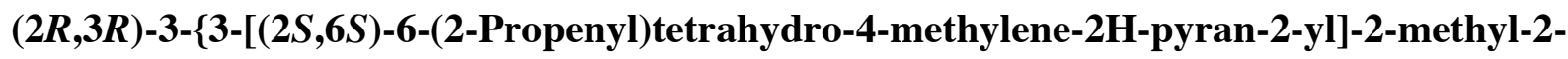
propenyl]oxirane methanol ( $7 \mathrm{~b})$

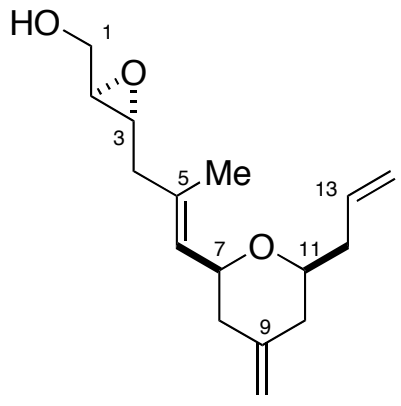

$7 b$

${ }^{1} \mathbf{H}$ NMR $\left(500 \mathrm{MHz}, \mathrm{CDCl}_{3}\right): \square 5.85$ (dddd, $\mathrm{J}=17.0,10.5,8.0$, and $\left.7.0 \mathrm{~Hz}, 1 \mathrm{H}, \mathrm{H}-13\right), 5.35$ (dq, $\mathrm{J}=7.5$ and $1.0 \mathrm{~Hz}, 1 \mathrm{H}, \mathrm{H}-6$ ), 5.10 (dddd, $\mathrm{J}=17.0,1.0,1.0$, and $1.0 \mathrm{~Hz}, 1 \mathrm{H}, \mathrm{H}_{\mathrm{cis}}-14$ ), 5.06 (dddd, $\mathrm{J}=10.0,2.0,1.0$, and $1.0 \mathrm{~Hz}, 1 \mathrm{H}, \mathrm{H}_{\text {trans }}-14$ ), 4.74 (app t, $\left.\mathrm{J}=1.5 \mathrm{~Hz}, 2 \mathrm{H}, \mathrm{H}_{2} \mathrm{C}=\mathrm{C}-9\right), 4.03$ (ddd, $\mathrm{J}$ $=11.0,8.0$, and $2.5 \mathrm{~Hz}, 1 \mathrm{H}, \mathrm{H}-7$ ), 3.94 (ddd, $\mathrm{J}=12.5,5.5$, and $\left.3.0 \mathrm{~Hz}, 1 \mathrm{H}, \mathrm{H}_{\mathrm{a}}-1\right), 3.66$ (ddd, $\mathrm{J}=$ 12.5, 7.5, and $4.0 \mathrm{~Hz}, 1 \mathrm{H}, \mathrm{H}_{\mathrm{b}}-1$ ), 3.38 (dddd, $\mathrm{J}=11.0,6.0,6.0$, and $2.5 \mathrm{~Hz}, 1 \mathrm{H}, \mathrm{H}-11$ ), 3.08 (ddd, $\mathrm{J}=5.5,5.5$, and $2.0 \mathrm{~Hz}, 1 \mathrm{H}, \mathrm{H}-3$ ), 2.95 (ddd, $\mathrm{J}=4.0,2.0$, and $2.0 \mathrm{~Hz}, 1 \mathrm{H}, \mathrm{H}-2$ ), 2.41 (ddddd, $\mathrm{J}=$ 14.0, 6.0, 6.0, 1.5, and $1.5 \mathrm{~Hz}, 1 \mathrm{H}, \mathrm{H}_{\mathrm{a}}-12$ ), $2.33\left(\mathrm{dd}, \mathrm{J}=14.5\right.$ and $\left.6.0 \mathrm{~Hz}, 1 \mathrm{H}, \mathrm{H}_{\mathrm{a}}-4\right), 2.29-2.16$ (m, 4H), 2.06 (app t, J = 12.5 Hz, 1H, $\left.\mathrm{H}_{\mathrm{ax}}-8\right), 1.94\left(\right.$ app t, J = 12.5 Hz, 1H, $\left.\mathrm{H}_{\mathrm{ax}}-12\right), 1.76$ (d, J = $1.0 \mathrm{~Hz}, 3 \mathrm{H}, \mathrm{Me}-\mathrm{C}-5$ ), and 1.61 (dd, $\mathrm{J}=7.5$ and $5.5 \mathrm{~Hz}, 1 \mathrm{H}, \mathrm{OH}$ ).

${ }^{13}$ C NMR (125 MHz, $\mathrm{CDCl}_{3}$ ): $\square$ 144.2, 135.0, 134.4, 128.0, 116.9, 108.7, 77.7, 75.4, 61.5, 58.3, $54.5,41.4,40.64,40.62,39.8$, and 17.3.

IR (neat): 3465, 3074, 2980, 2938, 2894, 1652, and $1419 \mathrm{~cm}^{-1}$.

HRMS (CI, $\mathbf{N H}_{3}$ in $\mathbf{C H}_{4}$ ) Calcd for $\mathrm{C}_{16} \mathrm{H}_{25} \mathrm{O}_{3}(\mathrm{M}+\mathrm{H})^{+}:$265.1804, found: 265.1794; Calcd for $\mathrm{C}_{16} \mathrm{H}_{29} \mathrm{NO}_{3}\left(\mathrm{M}+\mathrm{NH}_{4}\right)^{+}: 282.2069$, found: 282.2060 .

TLC $\mathrm{R}_{\mathrm{f}}=0.47$, hexanes : ethyl acetate $=1: 1$.

[ㅁ] ${ }_{D}^{23^{\circ} \mathrm{C}}=+18.0^{\circ}, \mathrm{c}=1.0, \mathrm{CH}_{2} \mathrm{Cl}_{2}$.

(2E,4Z)-5-Methyl-7-oxo-2,4-heptadienyl 2,2-dimethylpropanoate (8)<smiles>Cc1ccc(OCC=O)cc1</smiles> 
${ }^{1} \mathbf{H}$ NMR $\left(500 \mathrm{MHz}, \mathrm{CDCl}_{3}\right): \square 9.60(\mathrm{t}, \mathrm{J}=2.0 \mathrm{~Hz}, 1 \mathrm{H}, \mathrm{H}-1), 6.39(\mathrm{dd}, \mathrm{J}=15.0$ and $11.0 \mathrm{~Hz}, 1 \mathrm{H}$, $\mathrm{H}-5), 6.13(\mathrm{~d}, \mathrm{~J}=10.5 \mathrm{~Hz}, 1 \mathrm{H}, \mathrm{H}-4), 5.75$ (dt, J = 15.0 and $6.5 \mathrm{~Hz}, 1 \mathrm{H}, \mathrm{H}-6), 4.60$ (t, J = 6.5 Hz, 2H, H-7), 3.27 (d, J = 2.0 Hz, 2H, H-2), 1.87 (s, 3H, Me-C-3), and 1.21 (s, 9H, $t$-Bu-Pv). ${ }^{13} \mathrm{C}$ NMR $\left(125 \mathrm{MHz}, \mathrm{CDCl}_{3}\right)$ : $\square 198.2,178.1,129.9,128.65,128.63,127.1,64.5,47.6,38.7$, 27.1, and 24.7.

IR (neat): 3346, 2964, 1725, and $1480 \mathrm{~cm}^{-1}$.

TLC $R_{\mathrm{f}}=0.35$, hexanes : ethyl acetate $=6: 1$.

[(2E,4Z,8E)-10-[(2S,6S)-[2-[(1E)-3-[(2R,3R)-3-[[[(1,1-Dimethylethyl)dimethylsilyl]oxy]methyl]oxiranyl]-2-methyl-1-propenyl]tetrahydro-4-methylene-2Hpyran-6-yl]]-7-hydroxy-5-methyl-2,4,8-decatrienyl] 2,2-dimethylpropanoate (9)

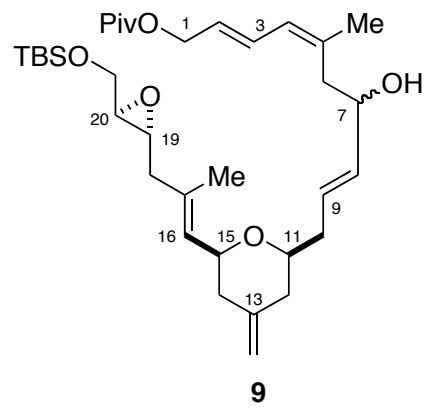

${ }^{1} \mathbf{H}$ NMR $\left(500 \mathrm{MHz}, \mathrm{CDCl}_{3}\right)$ : $\square 6.51(\mathrm{dd}, \mathrm{J}=15.0$ and $11.0 \mathrm{~Hz}, 1 \mathrm{H}, \mathrm{H}-3), 6.01(\mathrm{~d}, \mathrm{~J}=11.0 \mathrm{~Hz}$, $1 \mathrm{H}, \mathrm{H}-4), 5.73(\mathrm{dt}, \mathrm{J}=15.5$ and $6.6 \mathrm{~Hz}, 1 \mathrm{H}, \mathrm{H}-9), 5.67(\mathrm{dt}, \mathrm{J}=15.0$ and $6.6 \mathrm{~Hz}, 1 \mathrm{H}, \mathrm{H}-2), 5.57$ (dd, $\mathrm{J}=15.3$ and $6.4 \mathrm{~Hz}, 1 \mathrm{H}, \mathrm{H}-8), 5.34(\mathrm{dq}, \mathrm{J}=7.6$ and $1.0 \mathrm{~Hz}, 1 \mathrm{H}, \mathrm{H}-16), 4.74\left(\mathrm{~s}, 2 \mathrm{H}, \mathrm{H}_{2} \mathrm{C}=\mathrm{C}-\right.$ 13), 4.59 (m, 2H, $\left.\mathrm{H}_{\mathrm{a}, \mathrm{b}}-1\right), 4.25$ (m, 1H, H-7), 4.02 (ddd, $\mathrm{J}=10.8,8.6$, and $\left.2.3 \mathrm{~Hz}, 1 \mathrm{H}, \mathrm{H}-15\right), 3.77$ (dd, $\mathrm{J}=11.8$ and $\left.3.7 \mathrm{~Hz}, 1 \mathrm{H}, \mathrm{H}_{\mathrm{a}}-21\right), 3.70\left(\mathrm{dd}, \mathrm{J}=11.8\right.$ and $\left.4.7 \mathrm{~Hz}, 1 \mathrm{H}, \mathrm{H}_{\mathrm{b}}-21\right), 3.36$ (dddd, $\mathrm{J}=$ 11.2, 6.0, 6.0, and $2.4 \mathrm{~Hz}, 1 \mathrm{H}, \mathrm{H}-11$ ), 2.92 (ddd, J = 5.8, 5.8, and $2.1 \mathrm{~Hz}, 1 \mathrm{H}, \mathrm{H}-19), 2.87$ (ddd, J $=4.7,3.7$, and $2.1 \mathrm{~Hz}, 1 \mathrm{H}, \mathrm{H}-20), 2.54 / 2.53^{*}\left(\mathrm{dd}, \mathrm{J}=13.1\right.$ and $\left.8.4 \mathrm{~Hz}, 1 \mathrm{H}, \mathrm{H}_{\mathrm{a}}-6\right), 2.42-2.15(\mathrm{~m}$, 7H), $2.04\left(\mathrm{t}, \mathrm{J}=12.5 \mathrm{~Hz}, 1 \mathrm{H}, \mathrm{H}_{\mathrm{ax}}-14\right), 1.91$ (t, J = $\left.12.5 \mathrm{~Hz}, 1 \mathrm{H}, \mathrm{H}_{\mathrm{ax}}-12\right), 1.85$ (s, 3H, Me-C-5), 1.75 (d, J = 2.3 Hz, 3H, Me-C-17), 1.57 (d, J = 3.1 Hz, 1H, OH), 1.21 (s, 9H, $t$-Bu-Pv), 0.9 (s, $9 \mathrm{H}, t$-Bu-Si), 0.08 (s, 3H, Me-C-Si), and 0.07 (s, 3H, Me-C-Si).

${ }^{13}$ C NMR (125 MHz, $\left.\mathrm{CDCl}_{3}\right)$ : $\square$ 178.2, 144.3, 136.3, 135.3/135.2*, 134.7/134.6*, 129.7, 127.9, $127.57 / 127.53^{*}, 127.48,125.6,108.8,77.7,75.5,71.0,64.9,63.5,58.4,55.0,41.7,40.7$, 40.51/40.47*, 39.9, 39.15/39.10*, 38.7, 27.2, 25.9, 24.4, 18.3, 17.4, -5.3, and -5.4. IR (neat): 3470, 2957, 2934, 2898, 2858, 1729, 1653, and $1281 \mathrm{~cm}^{-1}$.

HRMS (ESI) Calcd for $\mathrm{C}_{35} \mathrm{H}_{58} \mathrm{O}_{6} \mathrm{SiNa}(\mathrm{M}+\mathrm{Na})^{+}: 625.3895$, found: 625.3888 . 
TLC $R_{\mathrm{f}}=0.45$, hexanes : ethyl acetate $=1: 1$.

[ए] ${ }_{D}^{23^{\circ} \mathrm{C}}=+6.4^{\circ}, \mathrm{c}=1.32, \mathrm{CH}_{2} \mathrm{Cl}_{2},(\mathrm{dr} .=2: 1)$.

* Due to two diastereomers.

[(2E,4Z,8E)-10-[(2S,6S)-[2-[(1E)-3-[(2R,3R)-3-[[[(1,1-Dimethylethyl)-

dimethylsilyl]oxy]methyl]oxiranyl]-2-methyl-1-propenyl]tetrahydro-4-methylene-2Hpyran-6-yl]]-7-[[(1,1-dimethylethyl)dimethylsilyl] oxy]-5-methyl-2,4,8-dectrienyl] 2,2dimethylpropanoate (10)

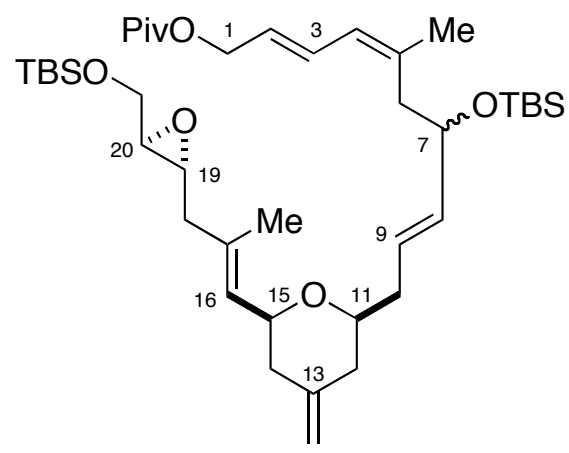

10

${ }^{1} \mathbf{H}$ NMR $\left(500 \mathrm{MHz}, \mathrm{CDCl}_{3}\right)$ : $\square 6.49(\mathrm{dd}, \mathrm{J}=15.0$ and $11.0 \mathrm{~Hz}, 1 \mathrm{H}, \mathrm{H}-3), 5.90(\mathrm{~d}, \mathrm{~J}=11.0 \mathrm{~Hz}$, 1H, H-4), 5.63-5.55 (m, 2H, H-2 and H-9), 5.48 (dd, J = 15.0 and 6.6 Hz, 1H, H-8), 5.33 (d, J = $7.5 \mathrm{~Hz}, 1 \mathrm{H}, \mathrm{H}-16), 4.72$ (br s, 2H, $\left.\mathrm{H}_{2} \mathrm{C}=\mathrm{C}-13\right), 4.57$ (app d, J = 6.5 Hz, 2H, $\left.\mathrm{H}_{\mathrm{a}, \mathrm{b}}-1\right), 4.19$ (app q, J $=6.5 \mathrm{~Hz}, 1 \mathrm{H}, \mathrm{H}-7), 4.01(\mathrm{ddd}, \mathrm{J}=10.6,7.9$, and $2.6 \mathrm{~Hz}, 1 \mathrm{H}, \mathrm{H}-15), 3.77(\mathrm{dd}, \mathrm{J}=11.6$ and 3.8 $\left.\mathrm{Hz}, 1 \mathrm{H}, \mathrm{H}_{\mathrm{a}}-21\right), 3.69\left(\mathrm{dd}, \mathrm{J}=11.8\right.$ and $\left.4.7 \mathrm{~Hz}, 1 \mathrm{H}, \mathrm{H}_{\mathrm{b}}-21\right), 3.32(\mathrm{dddd}, \mathrm{J}=12.3,6.0,6.0$, and 2.3 $\mathrm{Hz}, 1 \mathrm{H}, \mathrm{H}-11$ ), 2.91 (ddd, $\mathrm{J}=5.8,5.8$, and $2.1 \mathrm{~Hz}, 1 \mathrm{H}, \mathrm{H}-19$ ), 2.87 (ddd, $\mathrm{J}=4.7,3.7$, and $2.1 \mathrm{~Hz}$, $1 \mathrm{H}, \mathrm{H}-20), 2.44 / 2.43^{*}\left(\mathrm{dd}, \mathrm{J}=12.9\right.$ and $\left.6.6 \mathrm{~Hz}, 1 \mathrm{H}, \mathrm{H}_{\mathrm{a}}-6\right), 2.38-2.29(\mathrm{~m}, 2 \mathrm{H}), 2.27-2.13(\mathrm{~m}, 5 \mathrm{H})$, 2.03 (app t, J = 12.5 Hz, 1H, $\mathrm{H}_{\mathrm{ax}}-14$ ), 1.90 (app t, J = 12.5 Hz, 1H, $\mathrm{H}_{\mathrm{ax}}-12$ ), 1.80 (s, 3H, Me-C-5), 1.75 (d, J = 1.3 Hz, 3H, Me-17), 1.20 (s, 9H, $t$-Bu-Pv), 0.9 (s, 9H, $t$-Bu-TBS-C21), 0.86/0.85* (s, 9H, $t$-Bu-TBS-C7), 0.074 (s, 3H, Me-Si), 0.065 (s, 3H, Me-Si), and 0.002/-0.004* (m, 6H, Me$\mathrm{Si})$.

${ }^{13}$ C NMR (75 MHz, $\left.\mathrm{CDCl}_{3}\right)$ : $\square$ 178.2, 144.4, 137.2, 135.77/135.72*, 135.1/134.9*, 130.9, 127.96/127.92*, 126.6, 125.76/125.72* 124.3, 108.6, 77.94/77.87* $75.4,72.33 / 72.27^{*}, 65.1$, $63.5,58.4,55.0,41.7,41.6,40.7,39.9,39.0,38.7,27.2,25.8,24.9,18.3,18.1,17.3,-4.4,-4.9$, 5.3 , and -5.4 .

IR (neat): 2955, 2931, 2895, 2857, 1729, and $1254 \mathrm{~cm}^{-1}$. 
HRMS (FABMS PEG + NaI) Calcd for $\mathrm{C}_{41} \mathrm{H}_{72} \mathrm{O}_{6} \mathrm{Si}_{2} \mathrm{Na}(\mathrm{M}+\mathrm{Na})^{+}:$739.4765, found: 739.4760 .

TLC $\mathrm{R}_{\mathrm{f}}=0.36$, hexanes : ethyl acetate $=9: 1$.

$[\text { ㅁ] }]_{D}^{23^{\circ} \mathrm{C}}=-1.3^{\circ}, \mathrm{c}=1.67, \mathrm{CH}_{2} \mathrm{Cl}_{2}$.

* Due to two diastereomers.

\section{$(2 E, 4 Z, 8 E)-10-[(2 S, 6 S)$-[2-[(1E)-3-[(2R,3R)-3-[[[(1,1-Dimethylethyl)dimethyl-}

silyl]oxy]methyl]oxiranyl]-2-methyl-1-propenyl]tetrahydro-4-methylene-2H-pyran-6-yl]]-7[[(1,1-dimethylethyl)dimethylsilyl]oxy]-5-methyl-2,4,8-decatrien-1-ol (11)

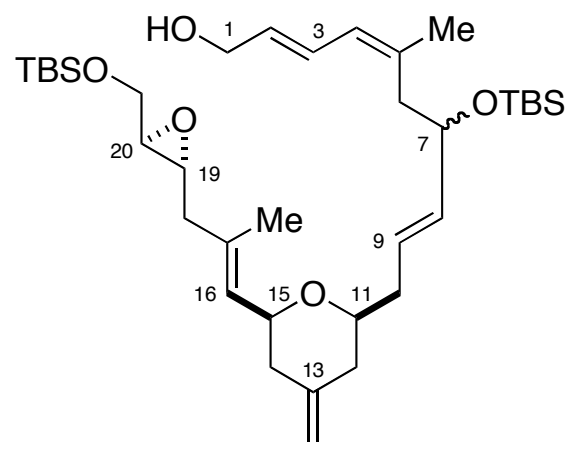

11

${ }^{1} \mathbf{H}$ NMR (500 MHz, $\left.\mathrm{CDCl}_{3}\right)$ : $\square 6.44(\mathrm{dd}, \mathrm{J}=15.0$ and $11.0 \mathrm{~Hz}, 1 \mathrm{H}, \mathrm{H}-3), 5.90(\mathrm{~d}, \mathrm{~J}=11.0 \mathrm{~Hz}$, 1H, H-4), 5.70 (dt, J = 15.0 and $6.0 \mathrm{~Hz}, 1 \mathrm{H}, \mathrm{H}-2), 5.59$ (dt, $\mathrm{J}=15.0$ and $7.0 \mathrm{~Hz}, 1 \mathrm{H}, \mathrm{H}-9), 5.48$ (dd, $\mathrm{J}=15.5$ and $6.5 \mathrm{~Hz}, 1 \mathrm{H}, \mathrm{H}-8$ ), 5.33 (dq, $\mathrm{J}=8.0$ and $1.0 \mathrm{~Hz}, 1 \mathrm{H}, \mathrm{H}-16$ ), $4.72(\mathrm{br} \mathrm{s}, 2 \mathrm{H}$, $\left.\mathrm{H}_{2} \mathrm{C}=\mathrm{C}-13\right), 4.20(\mathrm{ddd}, \mathrm{J}=6.5,6.5$, and $6.0 \mathrm{~Hz}, 1 \mathrm{H}, \mathrm{H}-7), 4.16\left(\mathrm{t}, \mathrm{J}=6.0 \mathrm{~Hz}, 2 \mathrm{H}, \mathrm{H}_{\mathrm{a}, \mathrm{b}}-1\right), 4.01$ (ddd, $\mathrm{J}=11.0,8.0$, and $3.0 \mathrm{~Hz}, 1 \mathrm{H}, \mathrm{H}-15$ ), 3.77 (dd, $\mathrm{J}=12.0$ and $4.0 \mathrm{~Hz}, 1 \mathrm{H}, \mathrm{H}_{\mathrm{a}}-21$ ), 3.69 (dd, $\mathrm{J}$ $=12.0$ and $\left.5.0 \mathrm{~Hz}, 1 \mathrm{H}, \mathrm{H}_{\mathrm{b}}-21\right), 3.32(\mathrm{~m}, 1 \mathrm{H}, \mathrm{H}-11), 2.91(\mathrm{ddd}, \mathrm{J}=6.0,6.0$, and $2.0 \mathrm{~Hz}, 1 \mathrm{H}, \mathrm{H}-$ 19), 2.87 (ddd, $\mathrm{J}=4.5,4.0$, and $2.0 \mathrm{~Hz}, 1 \mathrm{H}, \mathrm{H}-20$ ), 2.43 (ddd, $\mathrm{J}=13.0,7.0$, and $6.0 \mathrm{~Hz}, 1 \mathrm{H}, \mathrm{H}_{\mathrm{a}-}$ 6), 2.37-2.24 (m, 3H), 2.23-2.13 (m, 4H), $2.04\left(\mathrm{t}, \mathrm{J}=12.5 \mathrm{~Hz}, 1 \mathrm{H}, \mathrm{H}_{\mathrm{ax}}-14\right), 1.90(\mathrm{t}, \mathrm{J}=12.5 \mathrm{~Hz}$, $\left.1 \mathrm{H}, \mathrm{H}_{\mathrm{ax}}-12\right), 1.80$ (s, 3H, Me-C-5), 1.75 (d, J = 1.5 Hz, 3H, Me-C-17), 1.30 (t, J = 6.0 Hz, 1H, $\mathrm{OH}$ ), 0.90 (s, 9H, $t$-Bu-Si-C21), 0.87/0.86* (s, 9H, $t$-Bu-Si-C7), 0.074 (s, 3H, Me-Si-C21), 0.065 (s, 3H, Me-Si-C21), and 0.011/0.009/0.007* (s, 6H, Me-Si-C7).

${ }^{13}$ C NMR (75 MHz, $\mathrm{CDCl}_{3}$ ): $\square$ 144.5, 136.41/136.37*, 135.7/135.6* 135.2/135.1", 129.5, 128.72/128.70", 127.94/127.90*, 126.7, 125.74/125.69* $108.6,78.0 / 77.9^{*}, 75.5,72.4 / 72.3^{*}$, 
63.81/63.79", 63.5, 58.4, 55.0, 41.7, 41.6, 40.7, 39.94/39.89* 39.0, 25.9, 25.8, 24.97/24.95*, 18.3, $18.2,17.3,-4.4,-4.82 /-4.84^{*},-5.3$, and -5.4 .

IR (neat): 3446, 2953, 2929, 2857, 1472, and $1254 \mathrm{~cm}^{-1}$.

HRMS (ESI) Calcd for $\mathrm{C}_{36} \mathrm{H}_{64} \mathrm{O}_{5} \mathrm{Si}_{2} \mathrm{Na}(\mathrm{M}+\mathrm{Na})^{+}: 655.4190$, found: 655.4179 .

TLC $\mathrm{R}_{\mathrm{f}}=0.17$, hexanes : ethyl acetate $=5: 1$.

$[\square]]_{D}^{23^{\circ} \mathrm{C}}=-0.63^{\circ}, \mathrm{c}=1.47, \mathrm{CH}_{2} \mathrm{Cl}_{2},(\mathrm{dr} .=7: 6)$.

* Due to two diastereomers. 


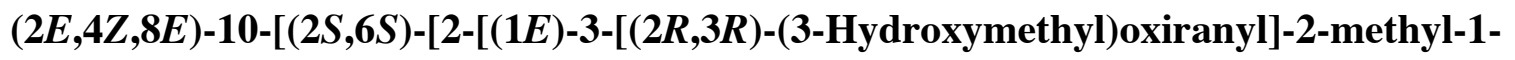
propenyl]tetrahydro-4-methylene-2H-pyran-6-yl]]-7-[[(1,1dimethylethyl)dimethylsilyl]oxy]-5-methyl-2,4,8-dectrienoic acid (12)

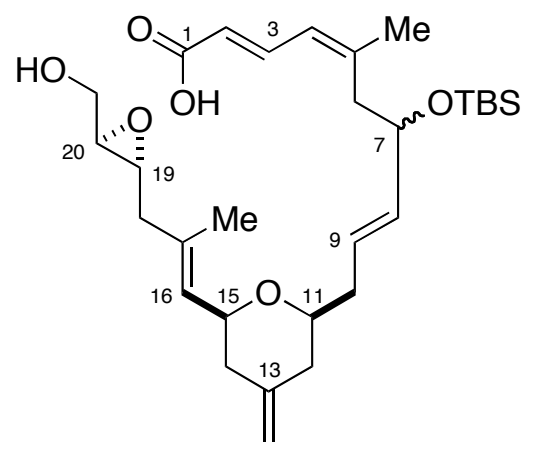

12

${ }^{1} \mathbf{H}$ NMR (500 MHz, CDCl $)$ : $\square 7.60(\mathrm{dd}, \mathrm{J}=15.5$ and $11.5 \mathrm{~Hz}, 1 \mathrm{H}, \mathrm{H}-3), 6.07(\mathrm{~d}, \mathrm{~J}=11.5 \mathrm{~Hz}$, 1H, H-4), 5.74 (d, J = 15.0 Hz, 1H, H-2), 5.69-5.62 (m, 1H, H-9), 5.51-5.46 (m, 1H, H-8), 5.34 (d, J = 7.5 Hz, 1H, H-16), 4.73 (s, 2H, $\mathrm{H}_{2} \mathrm{C}=\mathrm{C}-13$ ), 4.22 (m, 1H, H-7), 4.02/4.01* (ddd, J = 11.5, 8.0, and $3.5 \mathrm{~Hz}, 1 \mathrm{H}, \mathrm{H}-15$ ), 3.94 (ddd, $\mathrm{J}=13.0,2.5$, and $2.5 \mathrm{~Hz}, 1 \mathrm{H}, \mathrm{H}_{\mathrm{a}}-21$ ), 3.65 (ddd, $\mathrm{J}=12.5$, 4.0, and $2.5 \mathrm{~Hz}, 1 \mathrm{H}, \mathrm{H}_{\mathrm{b}}-21$ ), 3.341/3.335 (dddd, $\mathrm{J}=12.0,6.0,6.0$, and $2.5 \mathrm{~Hz}, 1 \mathrm{H}, \mathrm{H}-11$ ), 3.13/3.11* (ddd, $\mathrm{J}=7.0,5.0$, and $2.5 \mathrm{~Hz}, 1 \mathrm{H}, \mathrm{H}-19$ ), 2.98 (ddd, $\mathrm{J}=5.0,2.5$, and $2.5 \mathrm{~Hz}, 1 \mathrm{H}, \mathrm{H}-$ 20), 2.51 (ddd, $\mathrm{J}=13.0,7.5$, and $6.5 \mathrm{~Hz}, 1 \mathrm{H}, \mathrm{H}_{\mathrm{a}}-6$ ), 2.38 (ddd, $\mathrm{J}=13.5,5.0$, and $5.0 \mathrm{~Hz}, 1 \mathrm{H}, \mathrm{H}_{\mathrm{b}}-$ 6), 2.33-2.20 (m, 5H), 2.19-2.14 (m, 2H), 2.06-2.00 (m, 1H, $\left.\mathrm{H}_{\mathrm{ax}}-14\right), 1.96-1.90\left(\mathrm{~m}, 1 \mathrm{H}, \mathrm{H}_{\mathrm{ax}}-12\right)$, 1.91 (s, 3H, Me-C-5), 1.75 (s, 3H, Me-C-17), 0.85/0.84* (s, 9H, $t$-Bu-Si), and -0.009/-0.012/0.015/-0.017 (s, 6H, Me-Si).

${ }^{13}$ C NMR (75 MHz, $\left.\mathrm{CDCl}_{3}\right)$ : $\square 171.8,148.0 / 147.9^{*}, 144.3,142.8,135.5 / 135.3^{*}, 135.0 / 134.9^{*}$, $127.9,126.5 / 126.4^{*}, 125.72 / 125.66^{*}, 118.5,108.7,77.9 / 77.8^{*}, 75.4 / 75.3^{*}, 73.1,61.3 / 61.2^{*}, 58.7$, 54.9/54.8*, 42.1, 41.3, 40.7, 39.9/39.8*, 38.7, 26.01/25.94*, 25.8, 18.1, 17.5, -4.4, and -4.9.

IR (neat): 3410, 2952, 2931, 2893, 2856, 1685, 1635, and $1254 \mathrm{~cm}^{-1}$.

HRMS (ESI) Calcd for $\mathrm{C}_{30} \mathrm{H}_{48} \mathrm{O}_{6} \mathrm{SiNa}(\mathrm{M}+\mathrm{Na})^{+}: 555.3112$, found: 555.3102 .

TLC $\mathrm{R}_{\mathrm{f}}=0.33$, hexanes : ethyl acetate $=1: 1$ with $4 \% \mathrm{MeOH}$.

$[\square]_{D}^{23^{\circ} \mathrm{C}}=+23.9^{\circ}, \mathrm{c}=0.65, \mathrm{CH}_{2} \mathrm{Cl}_{2}$.

* Due to two diastereomers. 
$(1 S, 2 E, 5 S, 8 E, 10 Z, 14 E, 17 S)-5-[(1 R)-(1,2-D i h y d r o x y) e t h y l]-13-[[(1,1-$

dimethylethyl)dimethylsilyl]oxy]-3,11-dimethyl-19-methylene-6,21-

dioxabicyclo[15.3.1]heneicosa-2,8,10,14-tetraen-7-one (13)

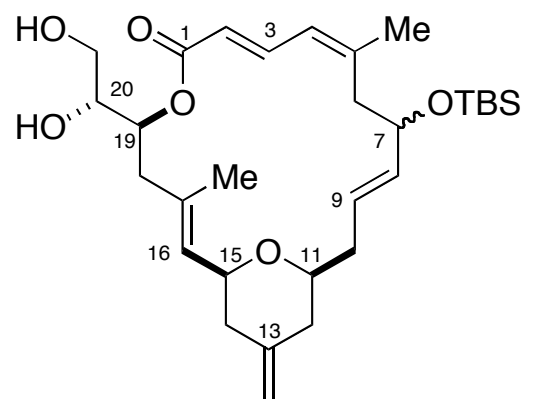

13

${ }^{1} \mathbf{H}$ NMR (500 MHz, $\left.\mathrm{CDCl}_{3}\right): \square 7.58 / 7.45^{*}(\mathrm{dd}, \mathrm{J}=15.0$ and $11.5 \mathrm{~Hz}, 1 \mathrm{H}, \mathrm{H}-3), 6.03 / 6.01^{*}(\mathrm{~d}, \mathrm{~J}=$ $11.0 \mathrm{~Hz}, 1 \mathrm{H}, \mathrm{H}-4), 5.75 / 5.69^{*}(\mathrm{~d}, \mathrm{~J}=15.0 \mathrm{~Hz}, 1 \mathrm{H}, \mathrm{H}-2), 5.65 / 5.58^{*}$ (dddd, $\mathrm{J}=15.5,7.5$, 6.0, and $1.5 \mathrm{~Hz}, 1 \mathrm{H}, \mathrm{H}-9), 5.46 / 5.44^{*}(\mathrm{dd}, \mathrm{J}=15.5$ and $6.5 \mathrm{~Hz}, 1 \mathrm{H}, \mathrm{H}-8), 5.31 / 5.26^{*}(\mathrm{~d}, \mathrm{~J}=8.0 \mathrm{~Hz}, 1 \mathrm{H})$, 5.09-5.04 (m, 1H, H-19), 4.71 (app s, 2H, H $2 \mathrm{C}=\mathrm{C}-13)$, 4.31-4.24 (m, 1H, H-7), 3.92 (ddd, J = 10.5, 8.0, and $2.5 \mathrm{~Hz}, 1 \mathrm{H}, \mathrm{H}-15), 3.76-3.65$ (m, 2H, $\mathrm{H}_{\mathrm{a}}-21$ and $\left.\mathrm{H}-20\right), 3.58$ (ddd, $\mathrm{J}=12.0,12.0$, and 5.0 Hz, $\left.1 \mathrm{H}, \mathrm{H}_{\mathrm{b}}-21\right), 3.37-3.27$ (m, 1H, H-11), 2.83/2.61* (dd, $\mathrm{J}=13.0$ and $\left.7.5 \mathrm{~Hz}, 1 \mathrm{H}, \mathrm{H}_{\mathrm{a}}-6\right)$, 2.51-2.37 (m, 3H), $2.27\left(\mathrm{dd}, \mathrm{J}=13.0\right.$ and $\left.6.5 \mathrm{~Hz}, 1 \mathrm{H}, \mathrm{H}_{\mathrm{b}}-6\right), 2.21-2.10$ (m, 4H), 2.04-1.91 (m, $2 \mathrm{H}), 1.94 / 1.88^{*}$ (s, 3H, Me-C-5), 1.74/1.68* (d, J = 1.5 Hz, 3H, Me-C-17), 0.89/0.87* (s, 9H, $t$ $\mathrm{Bu}-\mathrm{Si}$ ), and 0.040/0.036//0.024/0.006* (s, 6H, Me-Si).

${ }^{13}$ C NMR (75 MHz, $\left.\mathrm{CDCl}_{3}\right)$ : $\square 167.8 / 167.4^{*}, 148.4 / 147.5^{*}, 144.72 / 144.65^{*}, 141.8 / 141.4^{*}$, 133.7/133.3/132.3/129.9* $128.0 / 127.8^{*}, 125.5 / 125.2^{*}, 118.5 / 118.3^{*}, 108.53 / 108.48^{*}, 77.8 / 77.6^{*}$, $75.7 / 75.5^{*}, 73.0 / 72.4^{*}, 72.0 / 71.9^{*}, 71.2 / 70.9^{*}, 62.8 / 62.6^{*}, 41.4 / 41.2 / 40.8 / 40.62 / 40.56 / 40.53^{*}$, $39.1,38.5,25.9,24.9,18.3 / 18.1^{*}, 17.5 / 16.9^{*},-4.2,-4.5$, and -4.8 .

IR (neat): 3391, 2952, 2928, 2893, 2855, 1709, 1694, and $1256 \mathrm{~cm}^{-1}$.

HRMS (ESI) Calcd for $\mathrm{C}_{30} \mathrm{H}_{48} \mathrm{O}_{6} \mathrm{SiNa}(\mathrm{M}+\mathrm{Na})^{+}: 555.3112$, found: 555.3105 .

TLC $R_{\mathrm{f}}=0.31$, hexanes : ethyl acetate $=1: 1$.

$[\square]_{D}^{23^{\circ} \mathrm{C}}=+13.2^{\circ}, \mathrm{c}=0.49, \mathrm{CH}_{2} \mathrm{Cl}_{2},(\mathrm{dr}=1: 1)$.

* Due to two diastereomers. 
$(1 S, 2 E, 5 S, 8 E, 10 Z, 14 E, 17 S)-5$-[(1R)-(1,2-Dihydroxy)ethyl]-13-hydroxy-3,11-dimethyl-19methylene-6,21-dioxabicyclo[15.3.1] heneicosa-2,8,10,14-tetraen-7-one (14)

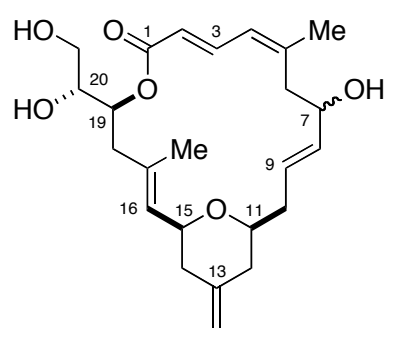

14

Spectral data of the less polar diastereomer.

${ }^{1} \mathrm{H}$ NMR (500 MHz, CDCl $)_{3}$ : $\square 7.56(\mathrm{dd}, \mathrm{J}=15.0$ and $11.6 \mathrm{~Hz}, 1 \mathrm{H}, \mathrm{H}-3), 6.06$ (d, J = $11.3 \mathrm{~Hz}$, 1H, H-4), 5.80 (d, J = 15.0 Hz, 1H, H-2), 5.73 (dddd, J = 15.7, 7.1, 5.9, and $1.3 \mathrm{~Hz}, 1 \mathrm{H}, \mathrm{H}-9$ ), 5.59 (dddd, $\mathrm{J}=15.8,4.9,1.3$, and $1.3 \mathrm{~Hz}, 1 \mathrm{H}, \mathrm{H}-8$ ), 5.26 (d, J = 8.0 Hz, 1H, H-16), 5.08 (ddd, J = 9.5, 7.0, and $2.7 \mathrm{~Hz}, 1 \mathrm{H}, \mathrm{H}-19), 4.72\left(\mathrm{br} \mathrm{s}, 2 \mathrm{H}, \mathrm{H}_{2} \mathrm{C}=\mathrm{C}-13\right.$ ), 4.34 (app t, dddd, $\mathrm{J}=6.3,6.3,6.3$, and $6.3 \mathrm{~Hz}, 1 \mathrm{H}, \mathrm{H}-7), 3.95$ (ddd, $\mathrm{J}=10.8,7.8$, and $2.5 \mathrm{~Hz}, 1 \mathrm{H}, \mathrm{H}-15), 3.73-3.67\left(\mathrm{~m}, 2 \mathrm{H}, \mathrm{H}_{\mathrm{a}}-21\right.$ and $\mathrm{H}-20), 3.60\left(\mathrm{~m}, 1 \mathrm{H}, \mathrm{H}_{\mathrm{b}}-21\right.$ ), 3.32 (dddd, $\mathrm{J}=11.3,9.6,2.2$, and $\left.2.2 \mathrm{~Hz}, 1 \mathrm{H}, \mathrm{H}-11\right), 2.81$ (dd, J $=13.4$ and $\left.7.9 \mathrm{~Hz}, 1 \mathrm{H}, \mathrm{H}_{\mathrm{a}}-6\right), 2.55(\mathrm{~d}, \mathrm{~J}=7.4 \mathrm{~Hz}, 1 \mathrm{H}, \mathrm{OH}-20), 2.45-2.41(\mathrm{~m}, 2 \mathrm{H}), 2.35(\mathrm{dd}, \mathrm{J}=$ 13.8 and $\left.9.3 \mathrm{~Hz}, 1 \mathrm{H}, \mathrm{H}_{\mathrm{b}}-6\right), 2.25-2.10(\mathrm{~m}, 5 \mathrm{H}), 2.01-1.93$ (m, 2H), 1.89 (s, 3H, Me-C-5), 1.71 (d, $\mathrm{J}=1.3 \mathrm{~Hz}, 3 \mathrm{H}, \mathrm{Me}-\mathrm{C}-17)$, and $1.43(\mathrm{~d}, \mathrm{~J}=5.9 \mathrm{~Hz}, 1 \mathrm{H}, \mathrm{OH}-7)$.

${ }^{13}$ C NMR (125 MHz, $\mathrm{CDCl}_{3}$ ): $\square 167.5,146.7,144.4,141.1,133.0,131.9,129.9,128.6,125.5$, 118.9, 108.6, 77.6, 75.9, 72.5, 71.2, 69.8, 62.8, 41.05, 41.00, 40.6, 40.1, 39.2, 24.1, and 16.9. TLC $\mathrm{R}_{\mathrm{f}}=0.32$, hexanes : ethyl acetate $=1: 2$, with $4 \% \mathrm{MeOH}$.

$[\text { [] }]_{D}^{23^{\circ} \mathrm{C}}=+6.0^{\circ}, \mathrm{c}=0.51, \mathrm{CH}_{2} \mathrm{Cl}_{2}$.

\section{Spectral data of the more polar diastereomer.}

${ }^{1} \mathbf{H}$ NMR (500 MHz, $\mathrm{CDCl}_{3}$ ): $\square 7.44(\mathrm{dd}, \mathrm{J}=15.3$ and $11.6 \mathrm{~Hz}, 1 \mathrm{H}, \mathrm{H}-3), 6.06(\mathrm{~d}, \mathrm{~J}=11.4 \mathrm{~Hz}$, 1H, H-4), 5.76 (d, J = 15.4 Hz, 1H, H-2), 5.67 (ddd, J = 15.4, 7.4, and 5.9 Hz, 1H, H-9), 5.61 (dd, $\mathrm{J}=15.4$ and $6.4 \mathrm{~Hz}, 1 \mathrm{H}, \mathrm{H}-8), 5.32(\mathrm{~d}, \mathrm{~J}=8.0 \mathrm{~Hz}, 1 \mathrm{H}, \mathrm{H}-16$ ), 5.05 (ddd, $\mathrm{J}=9.5,7.0$, and $2.7 \mathrm{~Hz}, 1 \mathrm{H}, \mathrm{H}-19$ ), 4.73 (br s, 2H, $\mathrm{H}_{2} \mathrm{C}=\mathrm{C}-13$ ), 4.25 (dddd, $\mathrm{J}=8.5,6.8,4.2$, and $3.7 \mathrm{~Hz}, 1 \mathrm{H}, \mathrm{H}-$ 7), 3.92 (ddd, $\mathrm{J}=10.4,7.9$, and $2.5 \mathrm{~Hz}, 1 \mathrm{H}, \mathrm{H}-15), 3.68-3.62\left(\mathrm{~m}, 2 \mathrm{H}, \mathrm{H}_{\mathrm{a}}-21\right.$ and $\mathrm{H}-20$ ), 3.58-3.53 (m, $1 \mathrm{H}, \mathrm{H}_{\mathrm{b}}-21$ ), 3.33 (dddd, $\mathrm{J}=11.4,9.1,2.5$, and $2.5 \mathrm{~Hz}, 1 \mathrm{H}, \mathrm{H}-11$ ), 2.60-2.51 (m, 4H), 2.44 $\left(\mathrm{dd}, \mathrm{J}=14.2\right.$ and $\left.9.5 \mathrm{~Hz}, 1 \mathrm{H}, \mathrm{H}_{\mathrm{a}}-18\right), 2.40(\mathrm{dd}, \mathrm{J}=8.8$ and $4.7 \mathrm{~Hz}, 1 \mathrm{H}, \mathrm{OH}-21), 2.27-2.23(\mathrm{~m}$, 
1H), 2.19-2.12 (m, 3H), 2.04-2.00 (m, 2H), 1.99 (s, 3H, Me-C-5), 1.67 (s, 3H, Me-C-17), and $1.52(\mathrm{~d}, \mathrm{~J}=3.8 \mathrm{~Hz}, 1 \mathrm{H}, \mathrm{OH}-7)$.

${ }^{13} \mathbf{C ~ N M R}\left(75 \mathrm{MHz}, \mathrm{CDCl}_{3}\right)$ : $\square 167.8,148.1,144.5,141.2,133.2,133.1,129.9,129.1,125.6$, $118.9,108.7,75.6,72.7,72.4,71.1,71.0,62.5,40.7,40.6,40.5,40.3,38.4,25.5$, and 16.6.

IR (neat): 3396, 2930, 1699, and $1278 \mathrm{~cm}^{-1}$.

HRMS (FAB, NaI) Calcd for $\mathrm{C}_{24} \mathrm{H}_{34} \mathrm{O}_{6} \mathrm{Na}(\mathrm{M}+\mathrm{Na})^{+}:$441.2253, found: 441.2278.

TLC $\mathrm{R}_{\mathrm{f}}=0.30$, hexanes : ethyl acetate $=1: 2$, with $4 \% \mathrm{MeOH}$.

[]. ${ }_{D}^{23^{\circ} \mathrm{C}}=+32.8^{\circ}, \mathrm{c}=0.39, \mathrm{CH}_{2} \mathrm{Cl}_{2}$.

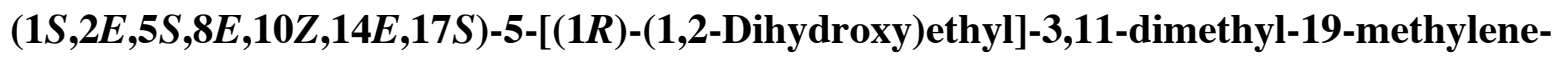
6,21-dioxabicyclo[15.3.1]heneicosa-2,8,10,14-tetraen-7,13-dione (15)

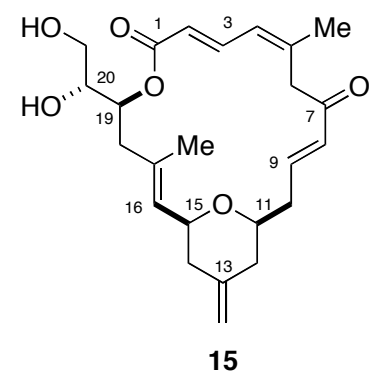

${ }^{1} \mathbf{H}$ NMR (500 MHz, $\mathrm{CDCl}_{3}$ ): $\square 7.63(\mathrm{dd}, \mathrm{J}=15.0$ and $11.5 \mathrm{~Hz}, 1 \mathrm{H}, \mathrm{H}-3), 6.83$ (ddd, J = 16.0, 9.0, and 4.5 Hz, 1H, H-9), 6.12 (d, J = 12.0 Hz, 1H, H-4), 5.95 (d, J = 15.5 Hz, 1H, H-8), 5.92 (d, $\mathrm{J}=15.0 \mathrm{~Hz}, 1 \mathrm{H}, \mathrm{H}-2), 5.20(\mathrm{~d}, \mathrm{~J}=8.0 \mathrm{~Hz}, 1 \mathrm{H}, \mathrm{H}-16), 5.13(\mathrm{ddd}, \mathrm{J}=11.0,7.0$, and $2.0 \mathrm{~Hz}, 1 \mathrm{H}$, H-19), 4.74 (app q, J = 1.5Hz, 2H, H $\mathrm{C}=\mathrm{C}-13), 4.10$ (d, J = 14.0 Hz, 1H, $\left.\mathrm{H}_{\mathrm{a}}-6\right), 3.97$ (ddd, J = 11.0, 8.0, and $2.5 \mathrm{~Hz}, 1 \mathrm{H}, \mathrm{H}-15), 3.76-3.63$ (m, 3H), 3.30 (dddd, $\mathrm{J}=12.0,10.0,2.0$, and $2.0 \mathrm{~Hz}$, 1H, H-11), 3.06 (d, J = 14.0 Hz, 1H, H and $\left.2.0 \mathrm{~Hz}, 1 \mathrm{H}, \mathrm{H}_{\mathrm{a}}-12\right), 2.29-1.91(\mathrm{~m}, 7 \mathrm{H}), 1.81(\mathrm{~s}, 3 \mathrm{H}, \mathrm{Me}-\mathrm{C}-5)$, and $1.71(\mathrm{~d}, \mathrm{~J}=1.0 \mathrm{~Hz}, 3 \mathrm{H}$, Me-C-17).

${ }^{13}$ C NMR (125 MHz, $\left.\mathrm{CDCl}_{3}\right)$ : $\square 197.9,167.0,146.3,143.63,143.57,140.0,132.6,131.3,129.6$, 125.3, 120.4, 109.1, 76.4, 75.9, 72.8, 70.9, 62.8, 45.1, 41.6, 40.9, 40.6, 40.1, 23.6, and 16.6. IR (neat): $3410,2935,2899,1700,1685,1635$, and $1281 \mathrm{~cm}^{-1}$.

HRMS (FABMS + NaI) Calcd for $\mathrm{C}_{24} \mathrm{H}_{32} \mathrm{O}_{6} \mathrm{Na}(\mathrm{M}+\mathrm{Na})^{+}: 439.2097$, found: 439.2081.

TLC $\mathrm{R}_{\mathrm{f}}=0.37$, hexanes : ethyl acetate $=1: 2$ with $2 \% \mathrm{MeOH}$.

$[\square]]_{D}^{23^{\circ} \mathrm{C}}=-76.8^{\circ}, \mathrm{c}=0.57, \mathrm{CH}_{2} \mathrm{Cl}_{2}$. 


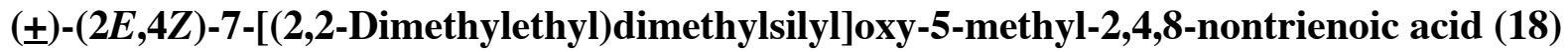

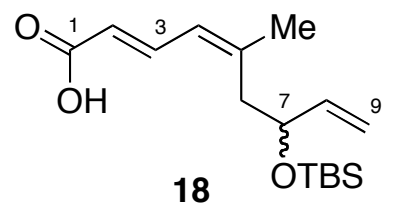

${ }^{1}$ H NMR (500 MHz, $\left.\mathrm{CDCl}_{3}\right): \square 7.65(\mathrm{dd}, \mathrm{J}=15.0$ and $11.5 \mathrm{~Hz}, 1 \mathrm{H}, \mathrm{H}-3), 6.11(\mathrm{~d}, \mathrm{~J}=11.5 \mathrm{~Hz}$, 1H, H-4), 5.81 (ddd, J = 17.5, 10.5, and $6.5 \mathrm{~Hz}, 1 \mathrm{H}, \mathrm{H}-8$ ), 5.77 (d, J = 15.0, 1H, H-2), 5.19 (ddd, $\mathrm{J}=17.0,2.0$, and $\left.1.0 \mathrm{~Hz}, 1 \mathrm{H}, \mathrm{H}_{\text {cis }}-9\right), 5.06\left(\mathrm{ddd}, \mathrm{J}=10.5,2.0\right.$, and $\left.1.0 \mathrm{~Hz}, 1 \mathrm{H}, \mathrm{H}_{\text {trans }}-9\right), 4.25$ (ddddd, $\mathrm{J}=8.0,6.5,5.5,1.5$, and $1.5 \mathrm{~Hz}, 1 \mathrm{H}, \mathrm{H}-7), 2.60\left(\mathrm{dd}, \mathrm{J}=13.0\right.$ and $\left.7.5 \mathrm{~Hz}, 1 \mathrm{H}, \mathrm{H}_{\mathrm{a}}-6\right)$, $2.36\left(\mathrm{dd}, \mathrm{J}=13.5\right.$ and $\left.4.8 \mathrm{~Hz}, 1 \mathrm{H}, \mathrm{H}_{\mathrm{b}}-6\right), 1.93$ (s, 3H, Me-C-5), 0.86 (s, 9H, $t$-Bu-Si), 0.014 (s, 3H, Me-Si), and 0.008 (s, 3H, Me-Si).

(500 MHz, DMSO-d6): $\square 12.03$ (br s, 1H, COOH), 7.39 (dd, $\mathrm{J}=15.0$ and $11.5 \mathrm{~Hz}, 1 \mathrm{H}, \mathrm{H}-3$ ), $6.14(\mathrm{~d}, \mathrm{~J}=11.5 \mathrm{~Hz}, 1 \mathrm{H}, \mathrm{H}-4), 5.86(\mathrm{ddd}, \mathrm{J}=16.0,10.5$, and $5.5 \mathrm{~Hz}, 1 \mathrm{H}, \mathrm{H}-8), 5.74(\mathrm{~d}, \mathrm{~J}=15.0$, $1 \mathrm{H}, \mathrm{H}-2), 5.22$ (d, J = 17.0 Hz, 1H, $\left.\mathrm{H}_{\text {cis }}-9\right), 5.06$ (d, J = $\left.10.5 \mathrm{~Hz}, 1 \mathrm{H}, \mathrm{H}_{\text {trans }}-9\right), 4.30$ (m, 1H, H-7), $2.53\left(\mathrm{dd}, \mathrm{J}=13.0\right.$ and $\left.8.5 \mathrm{~Hz}, 1 \mathrm{H}, \mathrm{H}_{\mathrm{a}}-6\right), 2.25\left(\mathrm{dd}, \mathrm{J}=13.5\right.$ and $\left.4.5 \mathrm{~Hz}, 1 \mathrm{H}, \mathrm{H}_{\mathrm{b}}-6\right), 1.89$ (s, 3H, Me-C-5), 0.82 (s, 9H, t-Bu-Si), -0.012(s, 3H, Me-Si), and -0.022 (s, 3H, Me-Si).

${ }^{13}$ C NMR (125 MHz, $\left.\mathrm{CDCl}_{3}\right)$ : $\square$ 173.1, 147.8, 143.4, 140.9, 126.0, 118.3, 114.3, 72.80, 41.78, 25.8, 25.7, 18.1, -4.6, and -5.0.

IR (neat): 2959, 2932, 2858, 1728, and $1428 \mathrm{~cm}^{-1}$.

HRMS (CI, $\mathbf{N H}_{3}$ in $\mathbf{C H}_{4}$ ) Calcd for $\mathrm{C}_{16} \mathrm{H}_{29} \mathrm{O}_{3} \mathrm{Si}(\mathrm{M}+\mathrm{H})^{+}$: 297.1886, found: 297.1882; Calcd for $\mathrm{C}_{16} \mathrm{H}_{32} \mathrm{NO}_{3} \mathrm{Si}\left(\mathrm{M}+\mathrm{NH}_{4}\right)^{+}:$314.2151, found 314.2146.

TLC $R_{\mathrm{f}}=0.25$, hexanes : ethyl acetate $=6: 1$. 


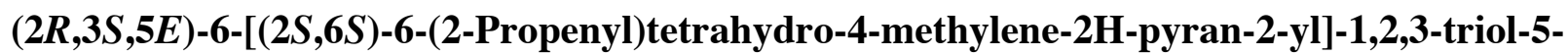
methyl-5-hexen-3-yl (2E,4Z)-7-[(2,2-dimethylethyl)dimethylsilyl]-oxy-5-methyl-2,4,8nontrienoate (19)

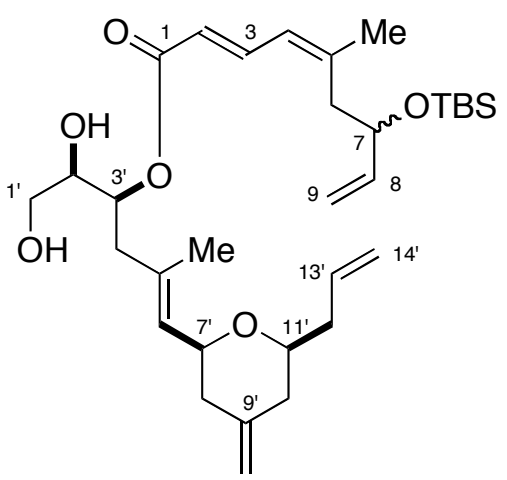

19

${ }^{1} \mathbf{H}$ NMR (500 MHz, $\left.\mathrm{CDCl}_{3}\right): \square 7.61 / 7.58^{*}(\mathrm{dd}, \mathrm{J}=15.5$ and $12.0 \mathrm{~Hz}, 1 \mathrm{H}, \mathrm{H}-3), 6.08(\mathrm{~d}, \mathrm{~J}=12.0$ Hz, 1H, H-4), 5.86-5.74 (m, 3H, H-2, H-8, and H-13'), 5.31 (d, J = 8.0 Hz, 1H, H-6'), 5.19 (dt, J $=17.0$ and $1.5 \mathrm{~Hz}, 1 \mathrm{H}), 5.09-4.98(\mathrm{~m}, 4 \mathrm{H}), 4.75$ (br s, 2H, $\left.\mathrm{H}_{2} \mathrm{C}=\mathrm{C}-9{ }^{\prime}\right), 4.24$ (m, 1H, H-7), 3.97 (ddd, $\mathrm{J}=11.0,7.5$, and $2.5 \mathrm{~Hz}, 1 \mathrm{H}, \mathrm{H}-7^{\prime}$ ) , 3.67-3.52 (m, 3H, $\mathrm{H}_{\mathrm{a}, \mathrm{b}}-1$ and $\left.\mathrm{H}-2\right), 3.33 / 3.32^{*}$ (dddd, J $=11.0,6.5,6.5$, and $\left.1.5 \mathrm{~Hz}, 1 \mathrm{H}, \mathrm{H}-11^{\prime}\right), 2.66-2.52(\mathrm{~m}, 4 \mathrm{H}), 2.39-2.31(\mathrm{~m}, 3 \mathrm{H}), 2.23-2.10(\mathrm{~m}$, 3H), 2.04-1.87 (m, 2H), 1.92 (s, 3H, Me-C-5), 1.72 (t, J = 1.5 Hz, 3H, Me-C-5'), 0.86 (s, 9H, $t$ Bu-TBS), and 0.009/0.008/-0.002/-0.004* (s, 6H, Me-TBS).

${ }^{13}$ C NMR (125 MHz, $\mathrm{CDCl}_{3}$ ): $\square 168.3,147.6,144.4,142.93 / 142.85^{*}, 140.9,134.8,134.6,128.8$, 126.0, 117.97/117.91* $116.8,114.3,108.6,77.6,75.4,72.97 / 72.96^{*}, 72.63,72.59,62.1$, 41.83/41.79*, 40.74, 40.71, 40.6, 39.9, 25.80/25.79*, 25.60/25.56* 18.1, 17.4, -4.6, and -4.9. IR (neat): $3428,2928,2856,1711,1637$, and $1278 \mathrm{~cm}^{-1}$.

HRMS (FAB, NaI) Calcd for $\mathrm{C}_{32} \mathrm{H}_{52} \mathrm{O}_{6} \mathrm{SiNa}(\mathrm{M}+\mathrm{Na})^{+}: 583.3431$, found: 583.3477.

TLC $R_{\mathrm{f}}=0.22$, hexanes : ethyl acetate $=2: 1$.

$[\square]_{D}^{23^{\circ} \mathrm{C}}=-29.8^{\circ}, \mathrm{c}=1.06, \mathrm{CH}_{2} \mathrm{Cl}_{2}$.

* Due to two diastereomers. 

dioxabicyclo-[15.3.1]-heneicosa-2,8,10,14-tetraen-5-yl]hydroxymethyl] 2,4-hexadienamide (21)

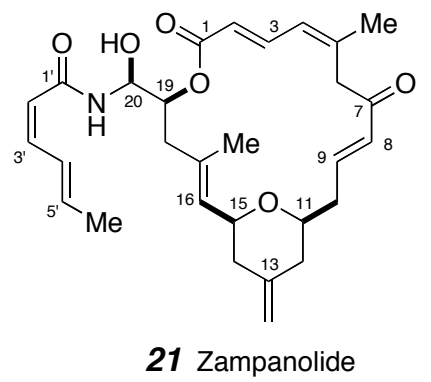

To a stirred solution of amidoalane 20 [prepared by addition of DIBAL-H (15 $\square \mathrm{L}, 1 \mathrm{M}$ in hexanes) to a THF $(0.4 \mathrm{~mL})$ solution of $2 Z, 4 E$-dienylamide $(\mathbf{9}, 2 \mathrm{mg}, 0.02 \mathrm{mmol})$ at room temperature and stirring for $30 \mathrm{~min}$ ] was added dactylolide ( $1 \mathrm{mg}, 2.6 \square \mathrm{mol}$ in $0.4 \mathrm{~mL}$ of THF). The resulting solution was stirred for $60 \mathrm{~min}$ at room temperature and tlc indicated consumption of starting material. Reaction was quenched by addition of aqueous Rochelle's salt $(1 \mathrm{~mL})$ and vigorously stirred until both layers became clear. After partition between $\mathrm{CH}_{2} \mathrm{Cl}_{2}$ and $\mathrm{H}_{2} \mathrm{O}$, the combined organic layer was washed with brine, dried over $\mathrm{Na}_{2} \mathrm{SO}_{4}$, and concentrated. The crude product was purified by HPLC $\left(\mathrm{SiO}_{2}\right.$, hexane:EtOAc $\left.=1: 1\right)$ to provide, in order of elution, zampanolide (21) and C(20)-epi-zampanolide (epi-21).

${ }^{1} \mathbf{H}$ NMR (500 MHz, DMSO-d6): $\square 8.31$ (d, J = $\left.8.9 \mathrm{~Hz}, 1 \mathrm{H}, \mathrm{NH}\right), 7.52(\mathrm{dd}, \mathrm{J}=14.9$ and $11.5 \mathrm{~Hz}$, 1H, H-3), 7.48-7.42 (m, 1H, H-4'), 6.74 (ddd, J = 16.0, 8.5, and 5.6 Hz, 1H, H-9), 6.37 (t, J = $11.5 \mathrm{~Hz}, 1 \mathrm{H}, \mathrm{H}-3$ '), 6.20 (d, J = 11.9 Hz, 1H, H-4), 6.13 (d, J = 5.2 Hz, 1H, OH), 6.03-5.92 (m, 1H, H-5'), 5.95 (d, J = 16.4 Hz, 1H, H-8), 5.93 (d, J = 15.0 Hz, 1H, H-2), 5.65 (d, J = 11.6 Hz, $1 \mathrm{H}, \mathrm{H}-2$ '), 5.32 (ddd, J = 9.0, 6.2, and $4.9 \mathrm{~Hz}, 1 \mathrm{H}, \mathrm{H}-20$ ), 5.10 (d, J = 7.8 Hz, 1H, H-16), 4.96 (ddd, $\mathrm{J}=10.1,6.1$, and $1.8 \mathrm{~Hz}, 1 \mathrm{H}, \mathrm{H}-19), 4.73$ (br s, $2 \mathrm{H}, \mathrm{CH}_{2}=\mathrm{C}-13$ ), 4.13 (d, J = $14.2 \mathrm{~Hz}, 1 \mathrm{H}$, $\mathrm{H}_{\mathrm{a}}-6$ ), 3.86 (ddd, $\mathrm{J}=11.1,7.8$, and $\left.2.5 \mathrm{~Hz}, 1 \mathrm{H}, \mathrm{H}-15\right), 3.00\left(\mathrm{~d}, \mathrm{~J}=14.3 \mathrm{~Hz}, 1 \mathrm{H}, \mathrm{H}_{\mathrm{b}}-6\right), 2.37-2.25$ (m, 3H), 2.17 (app d, J = 12.6 Hz, 1H, $\left.\mathrm{H}_{\mathrm{eq}}-14\right), 2.12-2.03(\mathrm{~m}, 2 \mathrm{H}), 1.90-1.81$ (m, 2H), 1.79 (dd, J $=6.7$ and $1.3 \mathrm{~Hz}, 3 \mathrm{H}, \mathrm{Me}-\mathrm{C}-5$ '), 1.74 (s, 3H, Me-C-5), and 1.61 (s, 3H, Me-C-17). [The resonance for $\mathrm{H}-11$ is apparently under the residual $\mathrm{CD}_{3} \mathrm{SOCHD}_{2}$ peak.]

${ }^{1} \mathbf{H}$ NMR (500 MHz, $\left.\mathrm{CDCl}_{3}\right)$ : $\square 7.65(\mathrm{dd}, \mathrm{J}=15.2$ and $11.7 \mathrm{~Hz}, 1 \mathrm{H}, \mathrm{H}-3), 7.42(\mathrm{~m}, 1 \mathrm{H}, \mathrm{H}-4$ '), 6.82 (ddd, $\mathrm{J}=15.8,9.3$, and $4.2 \mathrm{~Hz}, 1 \mathrm{H}, \mathrm{H}-9), 6.46$ (t, J = 11.5 Hz, 1H, H-3'), 6.31 (d, J = 7.8 $\mathrm{Hz}, 1 \mathrm{H}, \mathrm{NH}), 6.11(\mathrm{~d}, \mathrm{~J}=11.6 \mathrm{~Hz}, 1 \mathrm{H}, \mathrm{H}-4), 6.06$ (dq, J = 15.2 and $6.7 \mathrm{~Hz}, 1 \mathrm{H}, \mathrm{H}-5$ '), 5.95 (d, J $=15.0 \mathrm{~Hz}, 1 \mathrm{H}, \mathrm{H}-2), 5.94(\mathrm{~d}, \mathrm{~J}=16.5 \mathrm{~Hz}, 1 \mathrm{H}, \mathrm{H}-8), 5.46-5.43(\mathrm{~m}, 1 \mathrm{H}, \mathrm{H}-20), 5.44(\mathrm{~d}, \mathrm{~J}=11.4$ 
$\mathrm{Hz}, 1 \mathrm{H}, \mathrm{H}-2$ '), 5.30 (ddd, J = 11.1, 5.8, and 1.9 Hz, 1H, H-19), 5.20 (d, J = 7.8 Hz, 1H, H-16), 4.73 (br s, $2 \mathrm{H}, \mathrm{CH}_{2}=\mathrm{C}-13$ ), 4.13 (d, J = 13.7 Hz, 1H, $\left.\mathrm{H}_{\mathrm{a}}-6\right), 3.96$ (ddd, $\mathrm{J}=10.9,8.1$, and $2.5 \mathrm{~Hz}$, 1H, H-15), 3.56 (d, J = 3.9 Hz, 1H, OH), 3.29 (m, 1H, H-11), 3.04 (d, J = 13.7 Hz, 1H, H $\mathrm{b}_{\mathrm{b}}-6$ ), $2.42\left(\mathrm{~d}, \mathrm{~J}=13.7 \mathrm{~Hz}, 1 \mathrm{H}, \mathrm{H}_{\mathrm{a}}-18\right), 2.37$ (dddd, $\mathrm{J}=14.7,9.7$, 4.6, and 1.6 Hz, 1H, $\left.\mathrm{H}_{\mathrm{a}}-10\right), 2.24$ (app dd, $\mathrm{J}=14.9$ and $\left.10.1 \mathrm{~Hz}, 1 \mathrm{H}, \mathrm{H}_{\mathrm{b}}-10\right), 2.21\left(\mathrm{dd}, \mathrm{J}=13.7\right.$ and $\left.11.0 \mathrm{~Hz}, 1 \mathrm{H}, \mathrm{H}_{\mathrm{b}}-18\right), 2.14(\operatorname{app~d}, \mathrm{J}=$ $\left.13.1 \mathrm{~Hz}, 1 \mathrm{H}, \mathrm{H}_{\mathrm{eq}}-14\right), 2.09$ (app d, J = 13.1 Hz, $\mathrm{H}_{\mathrm{eq}}-12$ ), 1.97-1.90 (m, 2H, $\mathrm{H}_{\mathrm{ax}}-12$ and $\mathrm{H}_{\mathrm{ax}}-14$ ), 1.87 (dd, $\mathrm{J}=6.9$ and $1.8 \mathrm{~Hz}, 3 \mathrm{H}, \mathrm{Me}-\mathrm{C}-5$ ') 1.81 (s, 3H, Me-C-5), and 1.72 (s, 3H, Me-C-17).

HRMS (ESI) Calcd for $\mathrm{C}_{29} \mathrm{H}_{37} \mathrm{NO}_{6} \mathrm{Na}(\mathrm{M}+\mathrm{Na})^{+}:$518.2519, found: 518.2538.

TLC $R_{\mathrm{f}}=0.47$, hexanes $:$ ethyl acetate $=1: 1$, with $2 \% \mathrm{MeOH}$. 
$(2 Z, 4 E)-N-[(1 R)-[(1 S, 2 E, 5 S, 8 E, 10 Z, 14 E, 17 S)-3,11-D i m e t h y l-19-m e t h y l e n e-7,13-d i o x o-6,21-$ dioxabicyclo-[15.3.1]-heneicosa-2,8,10,14-tetraen-5-yl]hydroxymethyl] 2,4-hexadienamide C(20)-epi-Zampanolide (21)

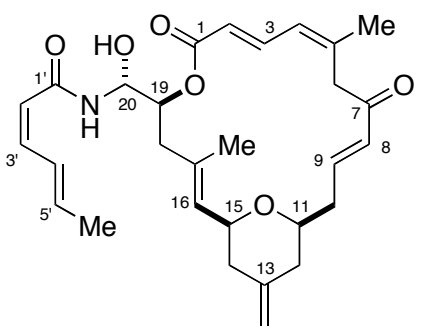

21-epi C(20)-epi-Zampanolide

${ }^{1}$ H NMR (500 MHz, DMSO-d6): $\square 8.41$ (d, J = 9.0 Hz, 1H, NH), 7.50 (dd, J = 14.8 and 11.6 Hz, 1H, H-3), 7.50-7.44 (m, 1H, H-4'), 6.74 (ddd, J = 16.3, 8.4, and 5.6 Hz, 1H, H-9), 6.42 (t, J = $11.3 \mathrm{~Hz}, 1 \mathrm{H}, \mathrm{H}-3$ '), 6.24 (d, J = 11.7 Hz, 1H, H-4), 6.03 (d, J = 4.9 Hz, 1H, OH), 6.02-5.97 (m, 1H, H-5'), 6.00 (d, J = 15.0 Hz, 1H, H-2), 5.93 (d, J = 16.2 Hz, 1H, H-8), 5.66 (d, J = 11.3 Hz, 1H, H-2'), 5.34 (ddd, J = 9.1, 5.9, and 4.9 Hz, 1H, H-20), 5.08 (d, J = 7.8 Hz, 1H, H-16), 5.03 (ddd, $\mathrm{J}=9.8,5.9$, and $2.6 \mathrm{~Hz}, 1 \mathrm{H}, \mathrm{H}-19), 4.73$ (br s, 2H, $\left.\mathrm{CH}_{2}=\mathrm{C}-13\right), 4.19$ (d, J = 14.0 Hz, 1H, $\left.\mathrm{H}_{\mathrm{a}}-6\right), 3.88$ (ddd, $\mathrm{J}=10.5,7.8$, and $\left.2.2 \mathrm{~Hz}, 1 \mathrm{H}, \mathrm{H}-15\right), 2.90$ (d, J = 14.1 Hz, 1H, H (m, 2H), 2.19-2.04 (m, 4H), 1.88-1.79 (m, 2H), $1.81(\mathrm{dd}, \mathrm{J}=6.9$ and 1.9 Hz, 3H, Me-C-5'), 1.75 (s, 3H, Me-C-5), and 1.63 (s, 3H, Me-C-17). [The resonance for $\mathrm{H}-11$ is apparently under the residual $\mathrm{CD}_{3} \mathrm{SOCHD}_{2}$ peak.]

${ }^{1} \mathbf{H}$ NMR (500 MHz, $\left.\mathrm{CDCl}_{3}\right)$ : $\square 7.67$ (dd, J = 15.0 and $\left.11.5 \mathrm{~Hz}, 1 \mathrm{H}, \mathrm{H}-3\right), 7.42$ (m, 1H, H-4'), 6.83 (ddd, $\mathrm{J}=16.4,9.4$, and $4.9 \mathrm{~Hz}, 1 \mathrm{H}, \mathrm{H}-9), 6.46$ (t, J = 11.5 Hz, 1H, H-3'), 6.34 (d, J = 7.9 Hz, 1H, NH), 6.13 (d, J = 11.4 Hz, 1H, H-4), 6.06 (dq, J = 14.3 and 7.0 Hz, 1H, H-5'), 6.00 (d, J $=15.0 \mathrm{~Hz}, 1 \mathrm{H}, \mathrm{H}-2), 5.94$ (d, J = $16.3 \mathrm{~Hz}, 1 \mathrm{H}, \mathrm{H}-8), 5.53$ (ddd, J = 7.7, 4.6, and $3.4 \mathrm{~Hz}, 1 \mathrm{H}, \mathrm{H}-$ 20), 5.45 (d, J = 11.2 Hz, 1H, H-2'), 5.27 (m, 1H, H-19), 5.25 (d, J = 8.5 Hz, 1H, H-16), 4.74 (br s, $\left.2 \mathrm{H}, \mathrm{CH}_{2}=\mathrm{C}-13\right), 4.12\left(\mathrm{~d}, \mathrm{~J}=13.8 \mathrm{~Hz}, 1 \mathrm{H}, \mathrm{H}_{\mathrm{a}}-6\right), 3.96(\mathrm{ddd}, \mathrm{J}=11.0,8.0$, and $2.8 \mathrm{~Hz}, 1 \mathrm{H}, \mathrm{H}-$ 15), 3.52 (d, J = 4.9 Hz, 1H, OH), 3.29 (m, 1H, H-11), 3.06 (d, J = 13.7 Hz, 1H, H $=13.9$ and $\left.11.0 \mathrm{~Hz}, 1 \mathrm{H}, \mathrm{H}_{\mathrm{a}}-18\right), 2.37$ (dddd, $\mathrm{J}=14.9$, 10.0, 4.9, and 2.2 Hz, 1H, $\left.\mathrm{H}_{\mathrm{a}}-10\right), 2.26$ (d, $\left.\mathrm{J}=13.8 \mathrm{~Hz}, 1 \mathrm{H}, \mathrm{H}_{\mathrm{b}}-18\right), 2.26-2.22\left(\mathrm{~m}, 1 \mathrm{H}, \mathrm{H}_{\mathrm{b}}-10\right), 2.15\left(\operatorname{app~d}, \mathrm{J}=13.3 \mathrm{~Hz}, 1 \mathrm{H}, \mathrm{H}_{\mathrm{eq}}-14\right), 2.10$ (app d, $\left.\mathrm{J}=13.4 \mathrm{~Hz}, 1 \mathrm{H}, \mathrm{H}_{\mathrm{eq}}-12\right), 1.99-1.91$ (m, 2H, $\mathrm{H}_{\mathrm{ax}}-12$ and $\mathrm{H}_{\mathrm{ax}}-14$ ), 1.88 (dd, $\mathrm{J}=6.8$ and 1.5 Hz, 3H, Me-C-5'), 1.82 (s, 3H, Me-C-5), and 1.72 (s, 3H, Me-C-17). 


\section{Copies of NMR Spectra}

${ }^{1} \mathrm{H}$ NMR $\left(\mathrm{CDCl}_{3}\right)$ Synthetic Dactylolide (1)

${ }^{13} \mathrm{C}$ NMR $\left(\mathrm{CDCl}_{3}\right)$ Synthetic Dactylolide (1) 25

${ }^{1} \mathrm{H}$ NMR (DMSO-d 6 ) Synthetic Zampanolide (21)

${ }^{1} \mathrm{H}$ NMR (DMSO-d ${ }_{6}$ ) Natural Zampanolide

(taken from Supporting Information for Smith, A. B., III;

Safonov, I. G.; Corbett, R. M. J. Am. Chem. Soc. 2001, 123, 12426-12427.)

${ }^{1} \mathrm{H}$ NMR $\left(\mathrm{CDCl}_{3}\right)$ Synthetic Zampanolide (21) 

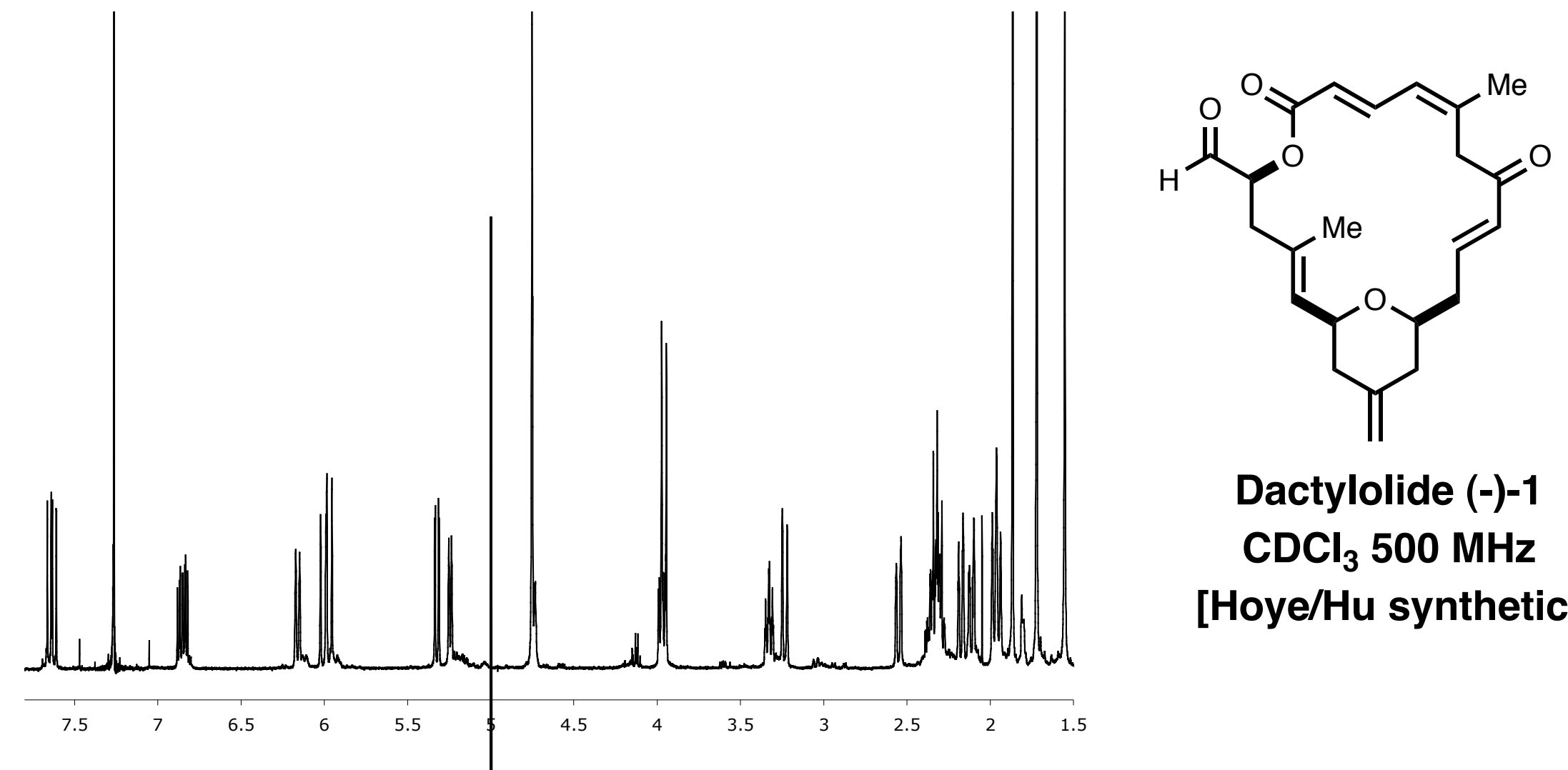

Dactylolide (-)-1 $\mathrm{CDCl}_{3} 500 \mathrm{MHz}$ [Hoye/Hu synthetic]

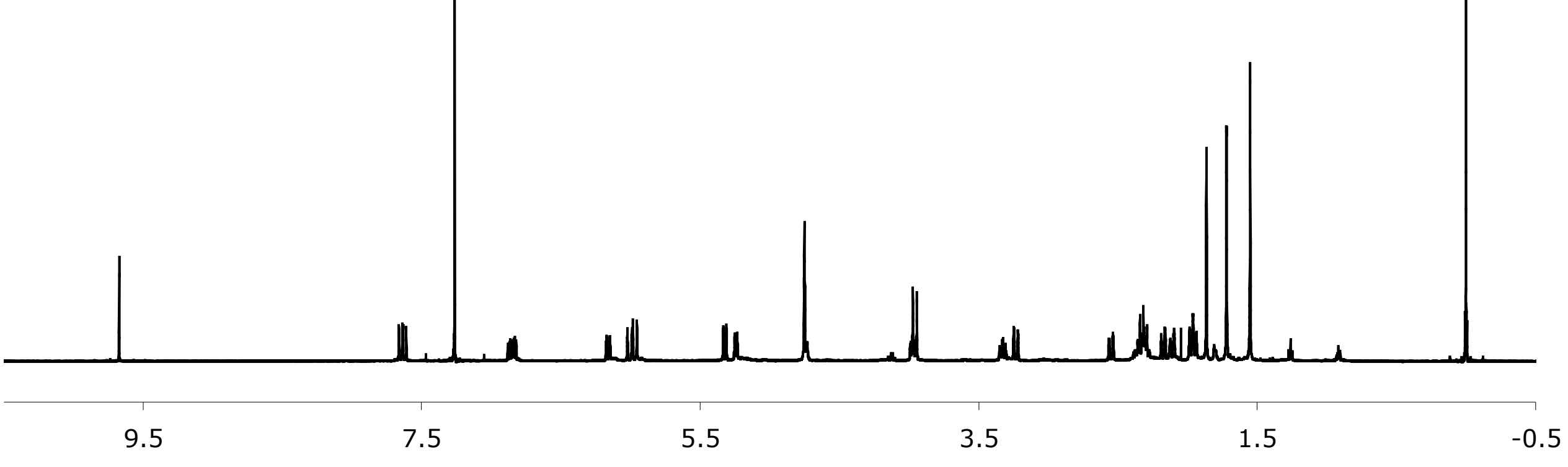




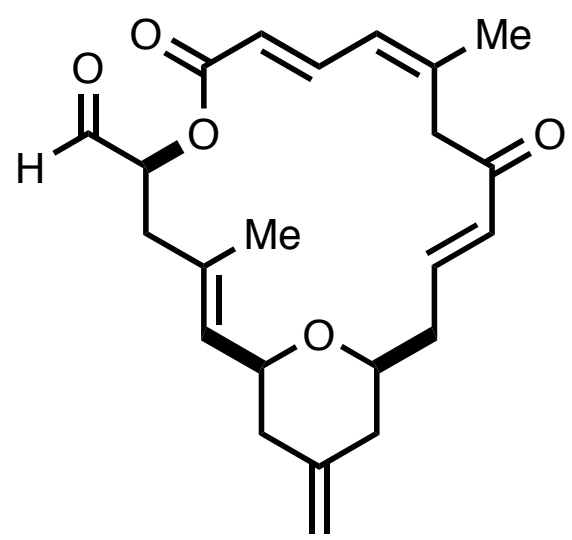

\section{Dactylolide (-)-1 $\mathrm{CDCl}_{3} 125 \mathrm{MHz}$}

[Hoye/Hu synthetic]

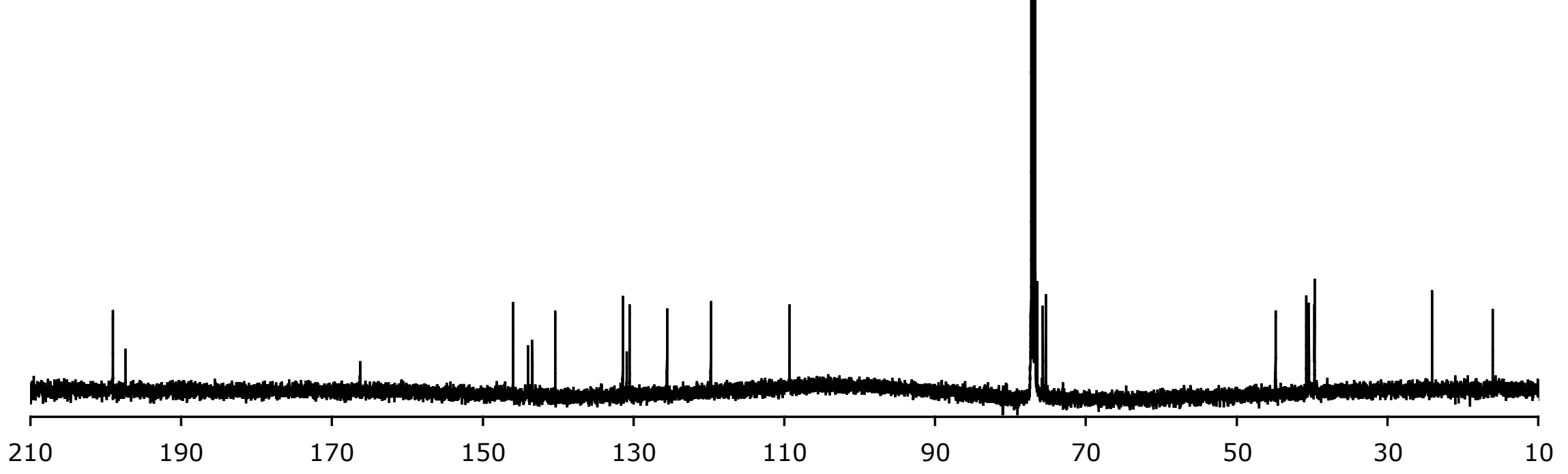




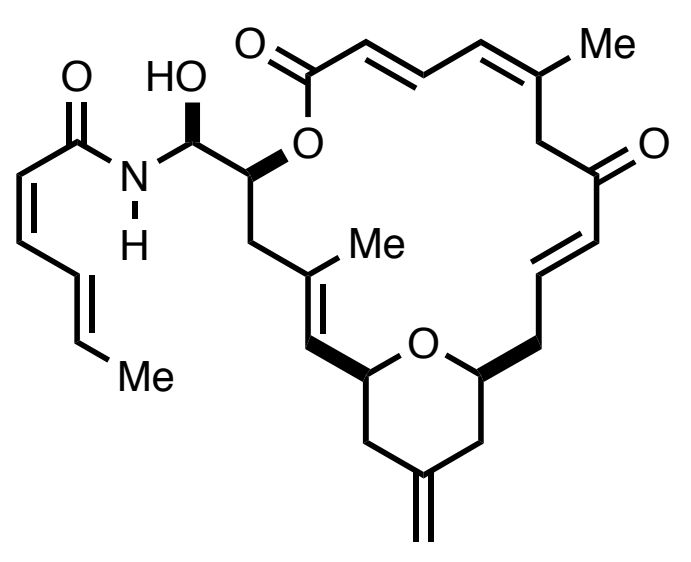

zampanolide (21) DMSO-d $_{6} 500 \mathrm{MHz}$ [Hoye/Hu synthetic] 


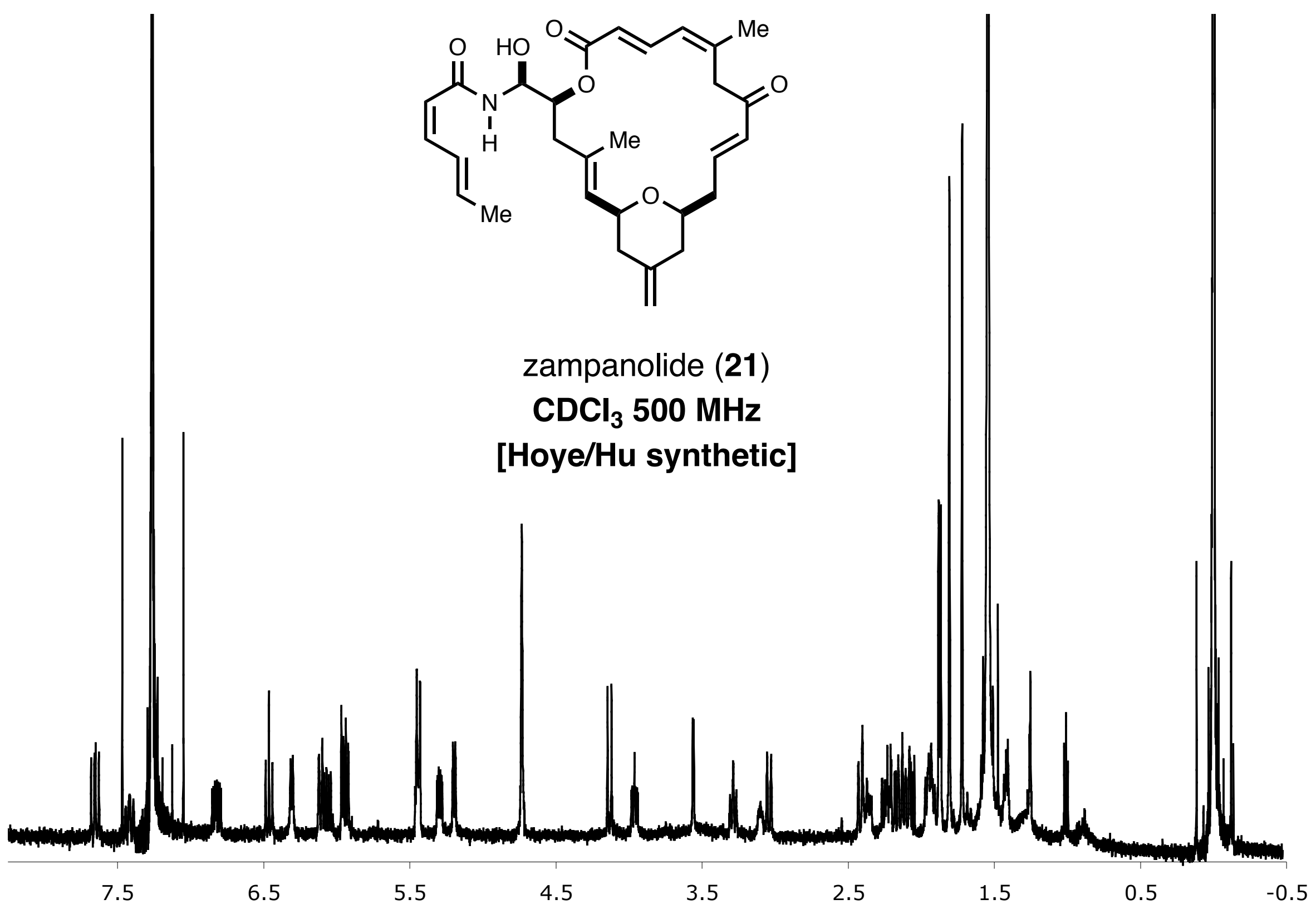




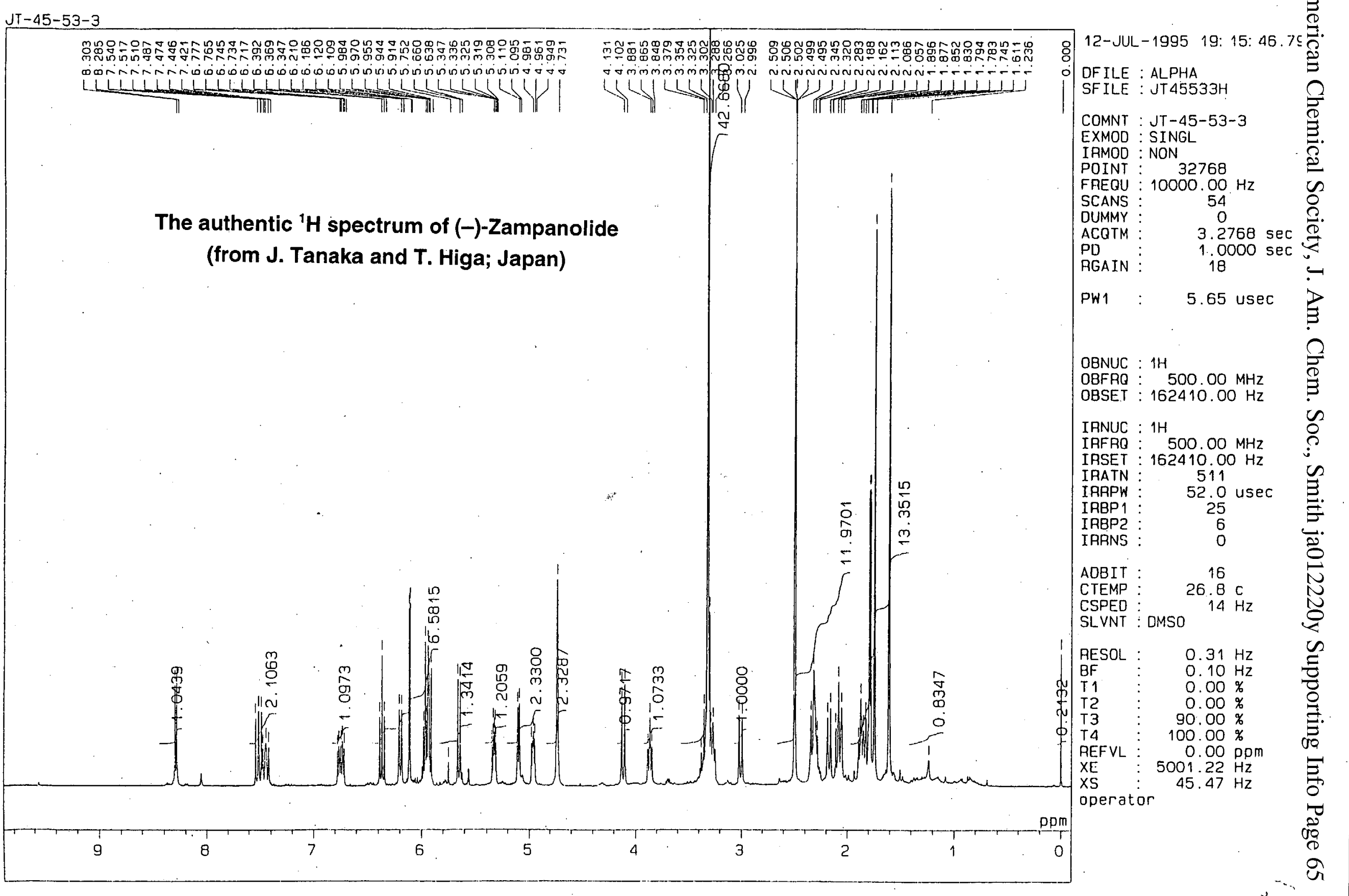




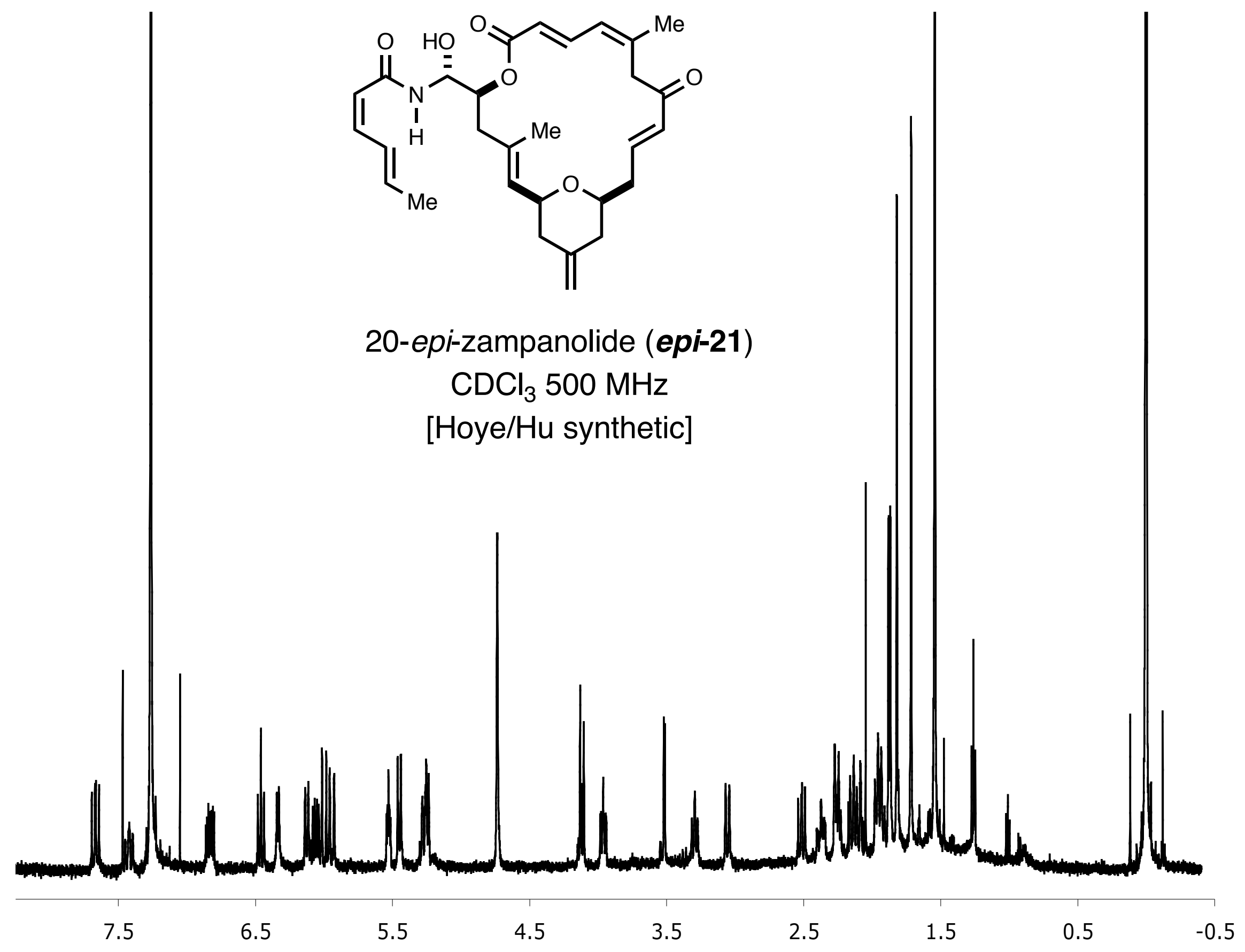

